Document downloaded from:

http://hdl.handle.net/10251/84432

This paper must be cited as:

Parra-Boronat, L.; Sendra, S.; Jimenez, JM.; Lloret, J. (2016). Multimedia sensors embedded in smartphones for ambient assisted living and e-health. Multimedia Tools and Applications. 75(21):13271-13297. doi:10.1007/s11042-015-2745-8

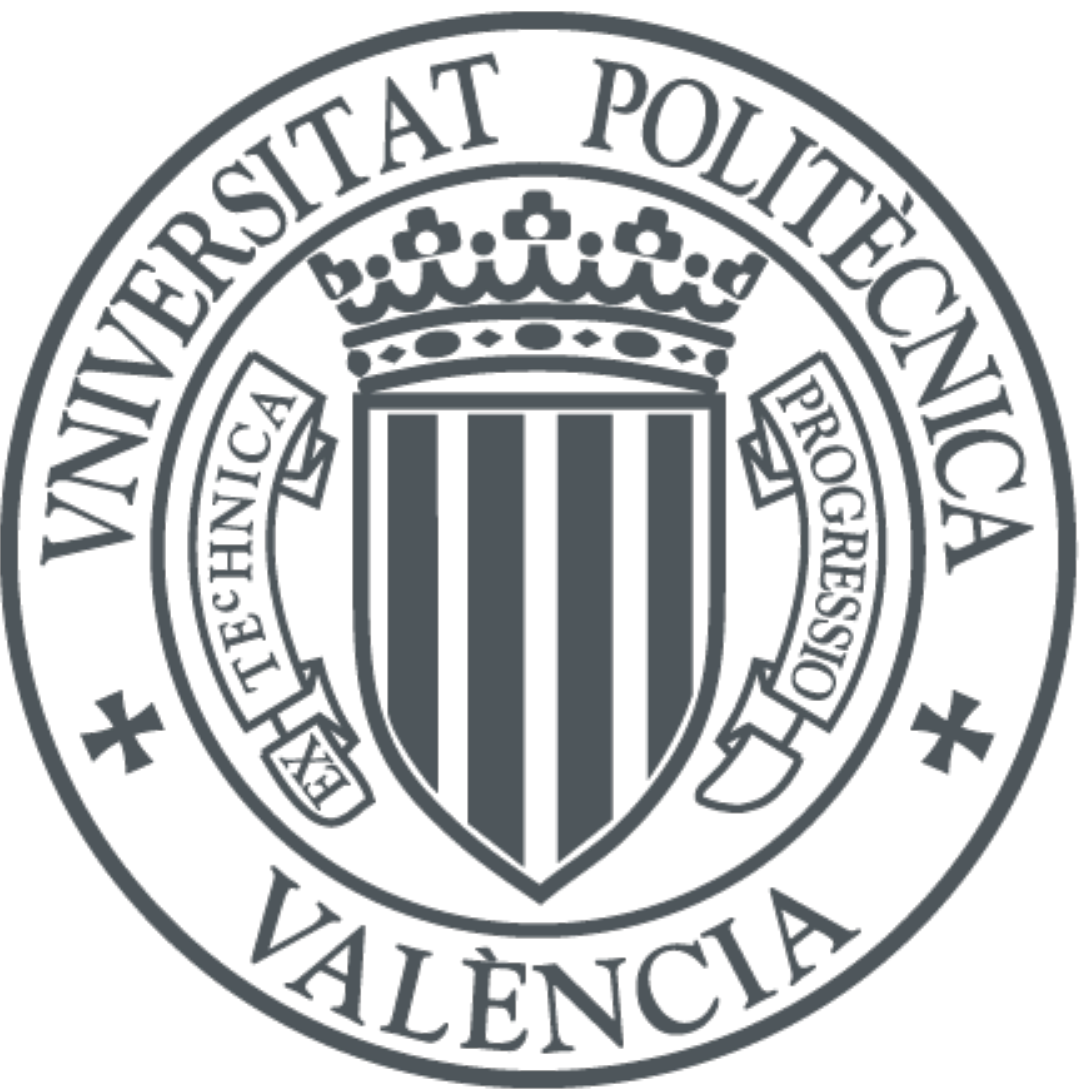

The final publication is available at

http://dx.doi.org/10.1007/s11042-015-2745-8

Copyright Springer Verlag (Germany)

Additional Information

The final publication is available at link.springer.com 


\section{Multimedia sensors embedded in smartphones for ambient assisted living and e-health}

Article in Multimedia Tools and Applications · July 2015

DOI: $10.1007 /$ s11042-015-2745-8

CITATIONS

5

4 authors:

\section{Lorena Parra}

Universitat Politècnica de València

23 PUBLICATIONS 74 CITATIONS

SEE PROFILE

\section{Jose M. Jimenez}

Universitat Politècnica de València

49 PUBLICATIONS 104 CITATIONS

SEE PROFILE

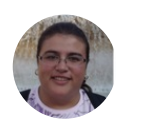

Sandra Sendra

University of Granada

102 PUBLICATIONS 872 CITATIONS

SEE PROFILE

\section{Jaime Lloret}

Universitat Politècnica de València

463 PUBLICATIONS 3,278 CITATIONS

SEE PROFILE

Some of the authors of this publication are also working on these related projects: 


\title{
Multimedia Sensors Embedded in Smartphones for Ambient Assisted Living and e-Health
}

\author{
Lorena Parra ${ }^{1}$, Sandra Sendra ${ }^{2}$, José Miguel Jiménez ${ }^{3}$, Jaime Lloret ${ }^{4}$ \\ Instituto de Investigación para la Gestión Integrada de zonas Costeras, Universidad Politécnica de \\ Valencia, 46730 Grao de Gandia (Valencia), Spain \\ ${ }^{1}$ loparbo@upvnet.upv.es, ${ }^{2}$ sansenco@posgrado.upv.es, ${ }^{3}$ jojiher@dcom.upv.es, ${ }^{4}$ jlloret@dcom.upv.es
}

\begin{abstract}
Nowadays, it is widely extended the use of smartphones to make human life more comfortable. Moreover, there is a special interest on Ambient Assisted Living (AAL) and eHealth applications. The sensor technology is growing and amount of embedded sensors in the smartphones can be very useful for AAL and e-Health. While some sensors like the accelerometer, gyroscope or light sensor are very used in applications such as motion detection or light meter, there are other ones, like the microphone and camera which can be used as multimedia sensors. This paper reviews the published papers focused on showing proposals, designs and deployments of that make use of multimedia sensors for AAL and ehealth. We have classified them as a function of their main use. They are the sound gathered by the microphone and image recorded by the camera. We also include a comparative table and analyze the gathered information.
\end{abstract}

Keywords: Multimedia content; Smartphone; AAL; e-health; Mobile sensing.

\section{Introduction}

Currently, the developed countries are suffering a generational change. The population is aging due to the low birthrate and the increase of life expectancy. This is mainly due to a change in their lifestyle and the advances in medicine. Nowadays in Europe, around $17 \%$ of the population is over 65 and it is expected that this percentage will reach $30 \%$ in 50 years [1]. Elderly people usually have several health afflictions. The $85 \%$ of them have at least one chronic disease while $65 \%$ suffer two or more chronic diseases [2]. The most common chronic diseases in elderly people are hypertension, heart diseases, eye disorders, arthritis and diabetes [2]. There are other impediments which are not directly related with chronic diseases and cause some troubles in the daily activities of elderly people. The losses of hearing, vision or mobility are also common problems in elderly people.

It is also important to know that most of elderly people ( $61 \%$ of men and $71 \%$ of women) live alone or with his/her old partner [3]. The ones who live alone generally have more accidents [4]. In fact, $30 \%$ of them have one fall per year and the $50 \%$ of them even suffer more than one fall. Elderly people generally are in touch with their family members via telephone calls and their families usually visit them once or twice per week [3]. The use of mobile phones by elderly people is still low. In United Kingdom, more than a half of the population between 65 and 75 years old has a mobile. However, this percentage decreases down to $30 \%$ in people over 75 years old [5]. The most frequent use of mobile phones by elderly people is limited to 
receive calls and make calls when there is an emergency. So, we can deduce that a great deal of elderly people is isolated of non-elderly people during most part of their time. If these persons suffered a fall and they could not get up from the floor by themselves, it could derive in many complications such as hypothermia, decubitus ulcer, pneumonia or even, in the worst cases, the death [4].

The concept of Ambient Assisted Living (AAL) was used for the first time in the European Framework Program for research funding. Its aim is to improve the Quality of Life (QoL) of people with special needs (like elderly and disabled people) by using different types of technology. AAL, and concretely Health researches, can be a promising help for elderly people by providing them more liberty, self-sufficiency and decrease the number of visits to the doctor. The main functions for AAL are focused on monitoring services, recognition of activities, people location [6], behavior detection and data collection [7]. To carry out these purposes, several sensors and smart devices are generally used. The data of these devices should be stored for further processing and for taking decisions when a problem has happened [8]. These devices can be placed at home (known as Smart Home), in the body (implementing wireless body area networks - WBANs), as wearable sensors, or even in the phones (Smartphones).

The sensors at home are very useful; they can benefit people when they are at home. One of the objectives of $\mathrm{H} 2020$ is to promote the active ageing, so it is expected that elderly people will not be all the time at home. The WBANs are incredible useful to monitor the patients' vital signs. However, there are some problems with this technology because some elderly people are reticent to wear them. The use of Smartphones to obtain information could help elderly people for preventing some dangerous situations. In addition, they can be used in indoors and outdoors and can be considered as non-annoying devices. The smartphones have several embedded sensors such as Global Position System (GPS), gyroscope, accelerometer, magnetometer, temperature sensor and some more ones. The camera and microphone integrated in smartphones are suitable to obtain information from the environment providing to the users the useful data for taking decisions in an intelligent ambient. The use of smartphones for sensing the environment can be considered as a new research field where several authors are developing new ideas. In this sense, E. Macias et al. presented a very complete survey [9] about mobile sensing. We can also find some works focused on adapting the mobile sensors to AAL and e-health applications. S. Sendra et al. proposed [10] a smart collaborative system for taking care of elderly and disabled people. The system uses the sensors embedded in the smartphones. Other smartphones applications, which try to endow intelligence to the environments, are the crowd control systems like the one developed in [11]. Finally, there exist other uses of mobile phone for AAL such as the predicting the position and tracking a person using its mobile. Several examples can be found in the literature [12-14].

Although nowadays, elderly people do not use the smartphones as desired, it is expected that in five years about $95 \%$ of people over 65 years will have one [15]. In the near future, the use of $5 \mathrm{G}$ will increase the smartphone functions as well as the communication speed to send and receive the data without undesired delays. This will also permit to send and receive higher amount of data. Although, the truth is that $5 G$ technology is not yet implemented, but there are several works about algorithms and protocols for $5 G$ phones [16]. One of main problems of 
smartphones is their low storage capacity. For this reason, there are several applications that give the possibility of storing the sensed information in the cloud. Sometimes, smartphones are not capable to create a cloud computing network and collaborate actively, but this problem was solved in [17] where R. Lacuesta et al. showed the design and deployment of a spontaneous ad-hoc cloud computing network for mobile devices. The energy consumption is also an important factor in the development of real implementations of AAL systems and eHealth applications. The smartphones have limitations in terms of energy and the wireless connections as $\mathrm{Wi}-\mathrm{Fi}$ and Bluetooth consume a considerable amount of energy. There are also some investigations focused on obtaining energy from the medium [18]. This is known as energy harvesting. There is a work that listed some energy efficiency indicators taking into account different VoIP codecs [19].

One of the main worries of elderly people about the use of smartphones for their monitoring is the security of the gathered data [20]. In this research field, several works were focused on creating secure protocols for mobile communications. An interesting proposal was developed by D. M. Monteiro et al. in [21], where they proposed a secure protocol for NFC-Bluetooth communications between mobile phones applied to cash credit transfer.

There are some studies carried out to know how elderly people will face to the use of smartphones. We can read in [15] that elderly people agree to wear a smartphone and use it, however, they are against replacing some medical visits by the use of smartphones $[22,23]$. These studies also point that all elderly people do not have the same physical conditions to use smartphones. People who have problems in manual dexterity or poor vision generally present more problems. In order to help them, there are several applications which use the multimedia sensors as the input information. It opens many possibilities to those people [24] with special needs. In fact, the use of the camera and microphone embedded in the smartphone are very common in other application areas. Cameras are used to recognize objects in museums, gesture recognition, document recognition and so on [25]. Microphone is used for speech recognition, for detecting the presence of conversation or even for detecting nonverbal cues. It is possible to use microphones for people localization according to the ambient noise [25] or even to recognize different kind of foods [26]. Another application based on the smartphone's camera is developed in [27]. In this case, during the record of a video frame, the application uses the GPS to tag the video with geo-localized information. According to [19], one of the main problems when using the smartphones' camera is that the smartphone usually remains in the pocket during long periods of time and the amount of data collected with the camera is very high to be stored in the smartphone. This problem was solved in [28], where E. Macias et al. proposed a semantic system where the video recorded by the mobile phone is tagged, so the video frames are sent jointly with some information about the place, environment, GPS position, etc. to a server in real time without high packet delay variations.

Although several surveys have been published about different kinds of technologies for AAL (like Wireless Sensor Networks (WSNs) [29], ambient intelligent techniques [30] for WBANs [31] and even for mHealth using mobile phones for elderly people), none of them mention the use of multimedia data [32]. There exist some surveys about the use of video records for activity recognition and behavior detection [33-35], but there is no one specific for 
smartphones. In the related literature, we have not found any survey about the use of multimedia data from Smartphones for AAL systems and e-health applications. Because different areas such medicine, informatics, telecommunications and computer science, are involved in the development of these applications, it is difficult to know the information about the number of available AAL and e-Health applications. For this reason, it is very important to perform this analysis. It can help technicians and researchers to manage multimedia data for AAL. Our survey is only focused on those works that use the embedded multimedia sensors in the smartphones. So, we do not take into account those works that include multimedia devices which are connected to the smartphones. This work is useful to compare the use of multimedia technology with other kind of systems developed for AAL and e-Health.

The rest of the paper is structured as follows. In order to show the utility of this survey, Section 2 shows some statistical data about the ages and gender using smartphones and the main health problems of the population. Section 3 reviews the published works that make use of the smartphones' camera for AAL and e-health purposes. Section 4 includes the review of the published works that make use of the smartphones' microphone for AAL and e-health purposes. A collection of different kinds of commercial Android applications that make use of the smartphones' camera or microphone are presented in Section 5. Section 6 compares and analyzes the works included in previous sections. Finally, Section 7 presents our conclusion and future work.

\section{Population with disabilities and the use of smartphones.}

In order to contextualize our study, it is important to know the evolution of the usage of the smartphones and which population sector is more affected by disabilities and chronic diseases. This section shows several statistics regarding to the use of mobile phones as a function of the population gender and their age. It also shows the percentage of smartphone operating systems.. Finally, we are going to show some interesting data about the most common diseases and disabilities.

According to a statistical study performed by the Pew Research Center's Internet \& American Life Project in 2013 [36], the number of men with mobile phones is higher than the women (See Figure 1), although they are quite close. In addition, we can see that the highest percentage of people using smartphones is between 18-24 and 25-34 age ranges (See Figure 2). In 2013, the most important fact was that the $18 \%$ of people with 65 and older own a smartphone. This measure was $13 \%$ in February 2012). 


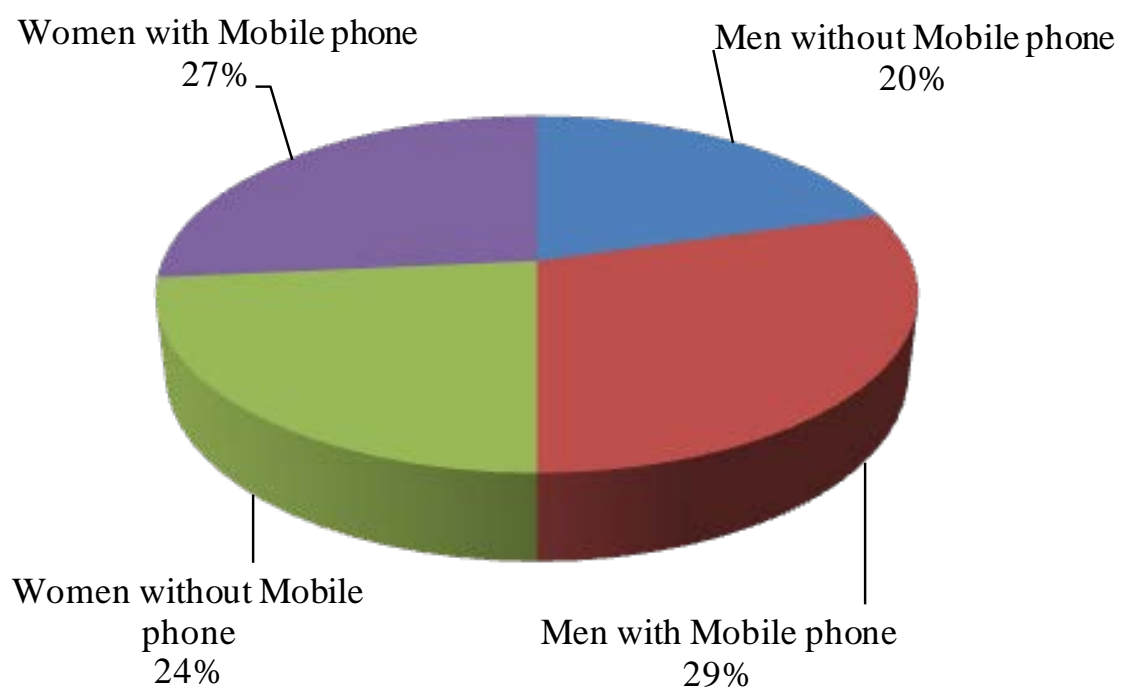

Figure 1. Use of mobile phones as a function of the population gender

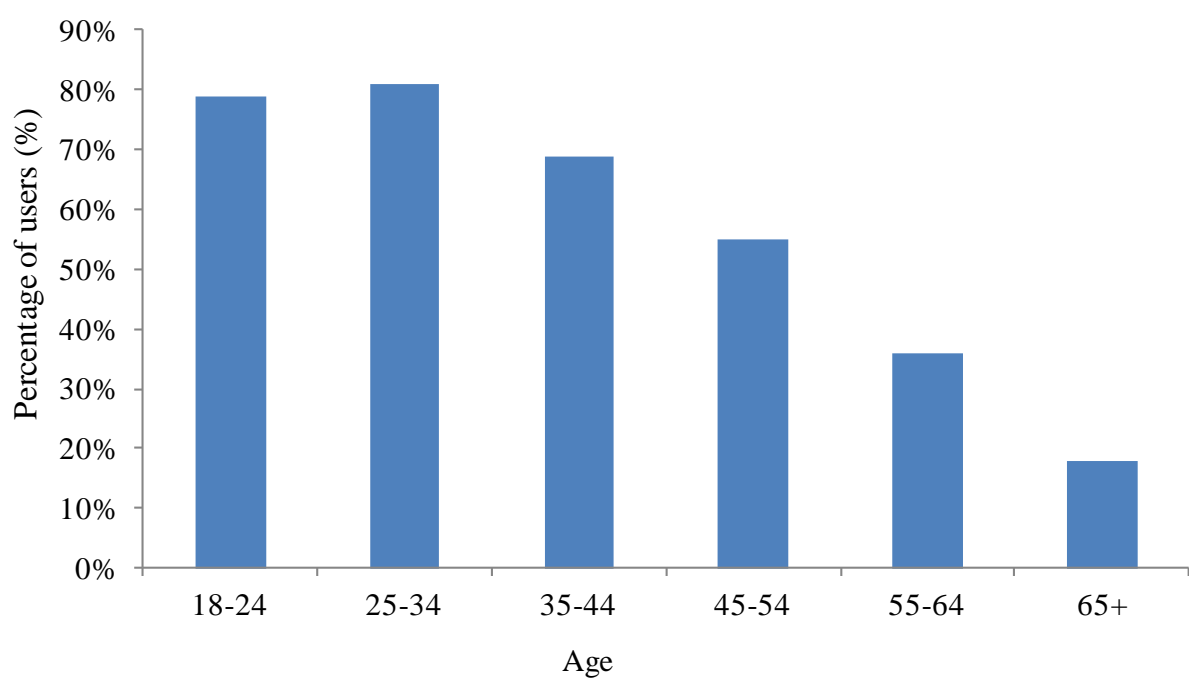

Figure 2. Population that uses mobile phones as a function of the population age

Regarding to the smartphone and its operating system (OS), there are currently four main OSs for smartphones and mobile phones: Android, Apple iOS, Windows Phone and Blackberry. There are other ones such as Symbian, but the number of users that nowadays choose this OS is very low. As we can see in Figure $3[37,38]$, Android is the most used OS $(52 \%)$ followed by Apple iOS (42\%). This is because Android is free and it is based on a modified version of the Linux kernel. This is an advantage for both the application developers and the users, because it permits customizing the phones in order to maximize their functions by simply installing an application. 


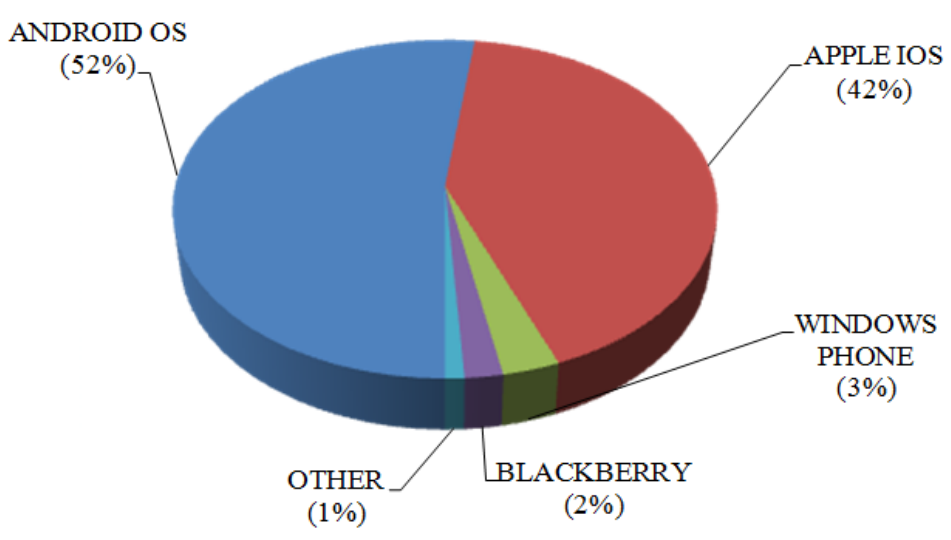

Figure 3. Operating Systems for mobile devices and smartphones

On the other hand, disabilities affect hundreds of million people in the developed countries. Around $10 \%$ of the total world's population, which are around 650 million people, is disabled. Figure 4 shows the relationship between the age of the population and the percentage of population that suffer some disability [39]. The highest percentage of disabled people is given in adults of 65 years old and older.

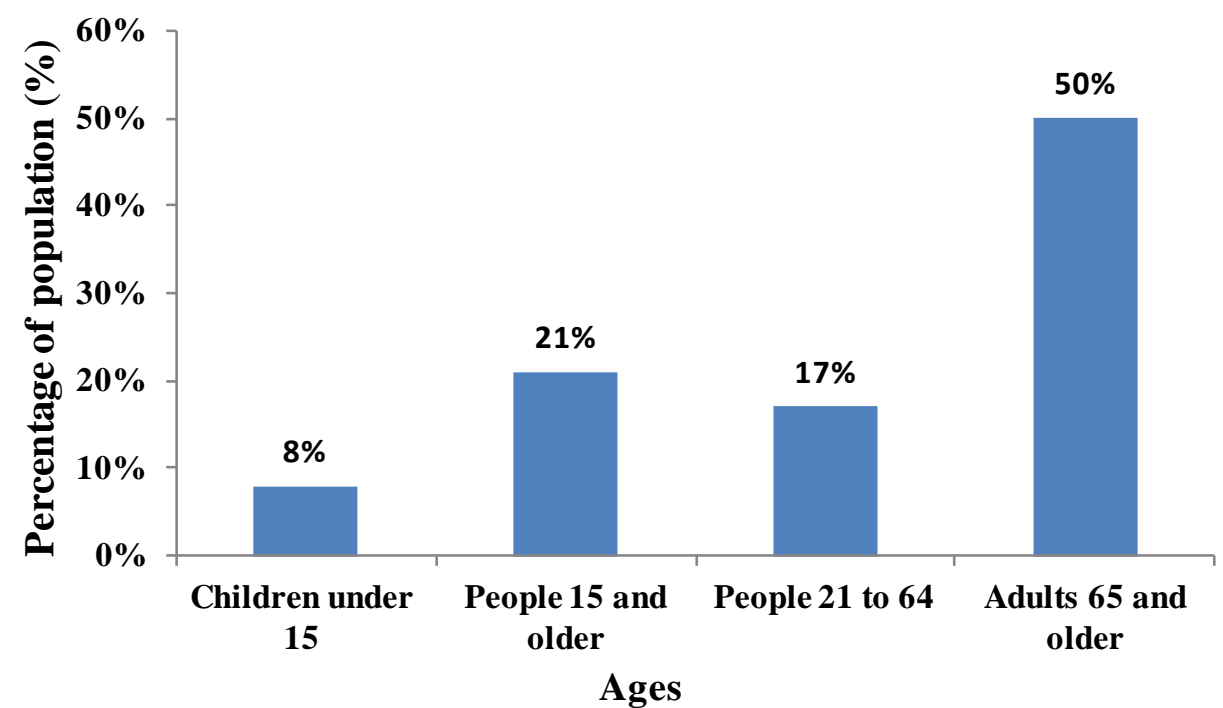

Figure 4. Distribution of population per ages that suffer some disability.

The main disabilities registered by the American population are shown in Figure 5 . As we can see, one of the main problems related to disabilities is the people mobility and their difficulty to carry out daily tasks. The highest percentage is registered for the difficulty of walking and/or going upstairs or downstairs. 


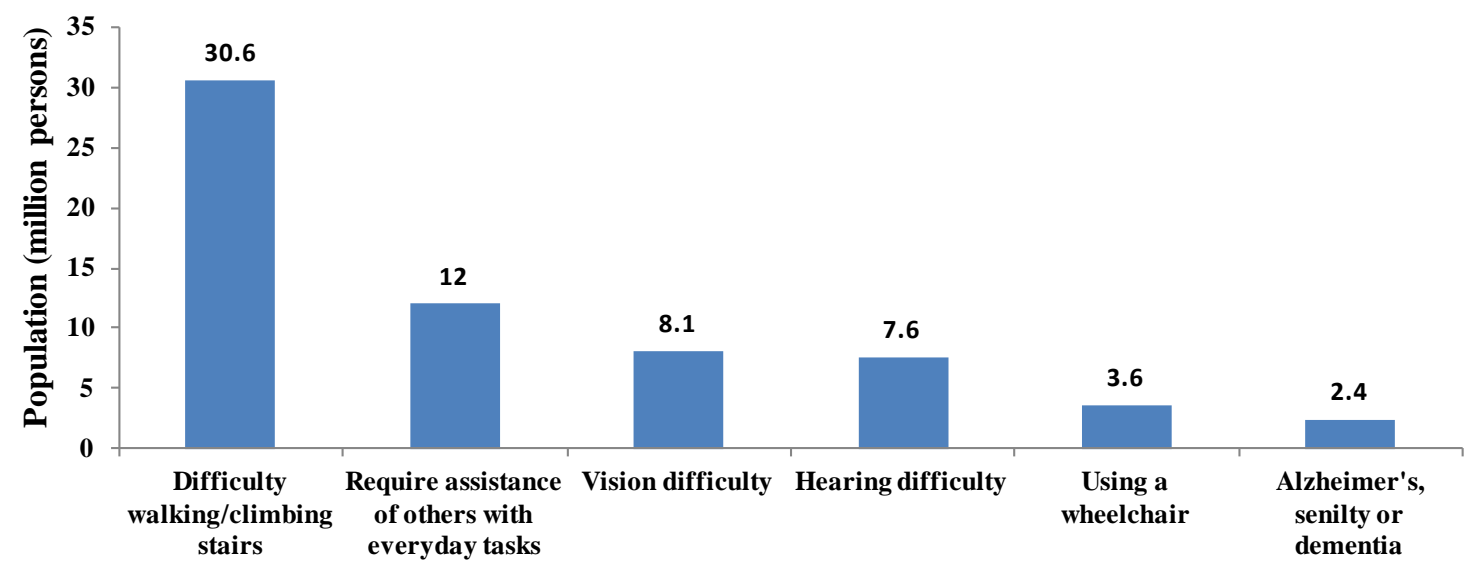

Figure 5. Main disabilities suffered by the population

Elderly people, who are increasing year by year, present needs that must be covered. According to the values shown in [3], the $81 \%$ of women and $78 \%$ of men follow a medical therapy and even need medical examination once or twice a month. In addition, elderly people frequently need medical assistance and, in general, they need to be hospitalized more than non-elderly people. They need several pharmacology products and sometimes they could need caregivers at home. The needs that should be covered are usually expensive, although sometimes they are partially financed by public services. In United Kingdom, the medical costs due to elderly people falls are almost $\$ 1.5$ billion every year [40]. The average health care spent per capita for people without chronic diseases is near to $\$ 800$. However, the cost for people with at least one chronic disease can reach $\$ 1,900$ every person. This value can rise up to $\$ 11,500$ when the number of diseases is five or more [2]. If the percentage of elderly people is increasing at this rate, in very few years this cost will be difficult to be assumed by some countries.

After analyzing the percentage of the population affected by chronic diseases and disabilities, we believe that the use of new technologies, in particular the use of multimedia sensors embedded in smartphones, can be a very useful tool to improve the autonomy and welfare of this population sector.

\section{Applications and proposals that use the smartphone's cameras.}

The evolution of smartphones and specially their video cameras are becoming more and more powerful which is fueling the emergence of new applications (some of them in e-health and AAL scenarios). Smartphones can be used in many daily tasks and nowadays these applications are suitable for monitoring the state of the health.

In this section, we are going to present several applications and research works where the camera of the smartphone is used. They are classified as a function of the parameter measured, providing the principle of operation for each case. 


\subsection{Heart Rate and cardiac diseases}

The principle of operation of the camera to gather data about heart rate is related to the changes of the skin color generated by the heartbeat. It can be done following next process. The LED on the phone illuminates part of the fingertip (as it is shown in Figure 6). The camera is able to capture the color changes and relates them to the beats per minute.

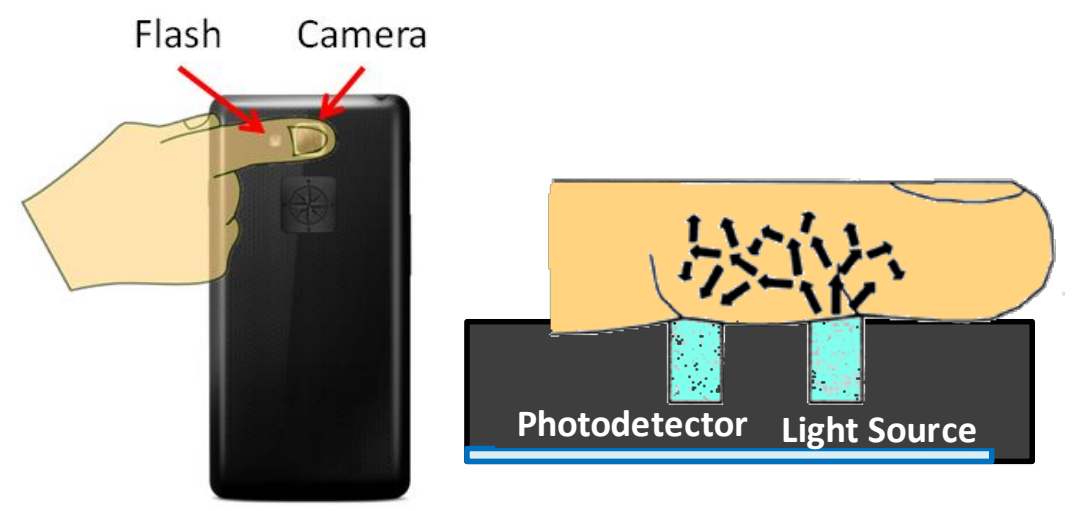

Figure 6 .Principle of operation

J. Lee et al. presented in [41] a smartphone application to measure the pulse time used to detect the Atrial Fibrillation (AF) in real time. The system is based on the use of the smartphone's camera which catches the heart rate fluctuations through the finger. The proposal does not require any additional hardware. The only requirement is to put the powerful LED, used as the camera flash, and the optical sensor, that is the camera, within fingertip. The application is developed for iPhone $4 \mathrm{~S}$ and tested with a set of 25 persons. The results are statistically analyzed following three statistical methods, statistical methods consisting of the Root Mean Square of Successive Differences (RMSSD), the Shannon entropy (ShE) and the Sample entropy (SampE), in order to assess heart rate variability. Authors have tried to achieve a sensitivity of $100 \%$ for iPhone data, reaching accuracies of $0.9844,0.8494$ and 0.9552 , respectively, for the three statistical methods.

D. Grimaldi et al. presented a solution to measure the photoplethysmography (PPG) with a smartphone's video camera in [42]. The system is a noninvasive way that measures the temporal variation of the blood volume. This parameter is normally measured with a pulse oximeter. Commercial devices use the light with red and infrared wavelengths which is passed through the subject to a photodetector. Because of the importance of this parameter, the application is able to detect any wrong usage of the system, i.e. when the finger is not placed correctly. The person should place the finger on the smartphone's camera in the way that it covers both the camera and the LED. The light passes through the finger and the camera detects the illumination changes. These changes are generated by the volumetric change of blood, for this reason, this method can be used to detect the PPG. To compute the PPG signal, the authors proposed the use of a threshold related to the number of pixels, although the authors advised that some other parameters such as different color saturation of the video, different finger's pressure on the camera or the movement of the finger respect to the camera should be considered. Authors confirmed the correctness and suitability of this application, comparing its results with the ones obtained by an oximeter. 
Heart rate variability is used to assess the autonomic nervous system (ANS) function. Normally, this parameter is measured through electrocardiograph (ECG), although there are problems to collect the big amounts of samples necessary for maintaining the statistical validity betweensubject psychophysiological comparisons. Bearing in mind these facts, J. A. J. Heathers [43] proposed an alternative to ECG which involves the use of an optical pulse sensor executed from a smartphone called smartphone pulse rate variability (SPRV). The author proposed a set of rules to take the measurements composed by periods of rests and controlled movements of arms and legs. Two experiments were performed to determine the accuracy between ECG and SPRV. SPRV values showed a consistent positive bias, which was successfully attenuated with correction. The system presents an accuracy of $\pm 2 \%$ for low-frequency spectral power, and $\pm 5 \%$ for high-frequency spectral power, which are acceptable values. Author concluded that this method has important advantages in terms of accessibility, portability and cost over traditional laboratory systems for the provision of cardiac psychophysiological data.

M. J. Gregoski et al. developed in [44] an Android application to measure Heart Rate (HR). The application is also based on the use of smartphone camera. The tests were performed by 14 subjects with ages between 20 and 58 where the HRs were collected simultaneously using the 3 devices during 5-minute periods while they were resting, reading aloud under observation, and playing a video game. The results of the test bench were compared with a Motorola Droid to ECG and Nonin 9560BT pulse oximeter readings during several movement-free tasks. The results indicate that the Android HR acquisition software provides valid HR measurements. Authors concluded that the lack of electrode patches or wireless sensor telemetric straps make this application easier to use than others. Moreover, from the patient point of view, this method can be considered as a non-invasive method which improves the promotion of preventive medicine programs.

\subsection{Defining of moods}

D. Lakens presented a new application to take heart rate measurements. In this case, he performed a study to demonstrate how smartphones are able to measure the PPG through the use of its digital camera. The results can be used to define the differences between heart rates as a function of relived emotional experiences [45]. The test was performed by seventy-four students (54 men, with an average age of 20) at the Eindhoven University of Technology. After performing the test, author discussed the possibility of measuring a person's HR using a video camera placed at a distance of several meters. Author concluded stating that his experiment demonstrates that smartphone PPG applications allow collecting reliable physiological data and it is possible to collect plethysmographic signals when the camera is positioned 0.5 to 1.5 meters away from the person.

\subsection{Diagnosis diseases using image processing}

Many applications and systems used to process images are based on artificial neural networks (ANNs). The process to design an efficient neural architecture is not an easy task because the 
final performance depends on the learning phase. In the previous step to the development and implementation of the system for visual pattern recognition, it is necessary to establish the characteristics of the input variables and the results to be achieved in the output. From these specifications, we can define both the learning phase and the test phase. Finally, the system must be validated depending on the results obtained. If they are not satisfactory, we should return to the design step to redefine some parameters. If the results are the desired ones, the system is ready to be used.

\subsubsection{Skin diseases diagnosis}

The key to quickly dealing the problems and skin diseases such as melanoma and skin cancer lies in the periodic review and early detection. For this reason, we can see a high emergence of applications running on a smartphone that are able to detect and catalog spots and freckles on the skin which could be harmful. The purpose of the application presented in [46] is to catalogue and categorize melanoma screening. However, in order to accept the validity of these e-Health tools, we should bear in mind some considerations such as the availability and accessibility of such tools, their costs, target audience, and, when possible, information on their efficacy.

A. Bourouis et al. [47] described an innovative development of a smartphone intelligent system used to analyze the skin images to identify cancer. The system uses the smartphone's camera and an ANN algorithm to process the images. The mobile device had high processing capacity to conduct the classification of skin images. The application is trained by using a MultiLayer Perceptron (MLP) where 100 pictures of abnormal skin and 100 normal cases were analyzed. The total error rate in this step was 0.00996279170 while the success rate to detect normal image from abnormal was $96.50 \%$. Authors highlighted in their conclusion that this system is a low cost mobile solution that can be used for patients who live in remote areas and for poor and developing countries, because skin examinations can be made at any place.

T. Wadhawan et al. proposed the use of a set of dermoscopic criteria widely used by clinicians for melanoma detection and the Implementation of the 7-point checklist for melanoma detection on smart devices [48]. In this case, authors use a smartphone to implement their proposal. Authors implemented an image processing system and pattern recognition algorithms that are able to run with the limited resources of a smartphone. The application is able to process the images in quasi real time to provide the classification results. Results show that when this application is combined with a commercial dermoscope that provides proper lesion illumination, the proposed system offers a truly self-contained handheld system for melanoma detection. Authors concluded that these devices could be used in a clinical setting for routine skin screening, or as low cost and portable assistive diagnostic device in underserved areas. It can also be used in developing countries with limited healthcare infrastructure.

\subsubsection{Retinal diseases diagnosis}


There are some diseases such as diabetes and cataract that can generate retinal blindness for millions of people. The current methods for obtaining retinal images are expensive and, sometimes, very problematic because these devices are not portable and it is needed a specialist to manage them.

Considering these drawbacks, A. Bourouis et al. [49] present an innovative system based on a low cost smartphone with an integrated microscopic lens. The mobile diagnosis application uses an ANN algorithm for capturing retinal images and analyzing and identifying possible retinal diseases. Authors developed the system for the Android operating platform. The proposal is tested and verified using two medical ophthalmology databases for demonstrating its capabilities. Results show that the system provides competitive retinal disease detection accuracy rates bigger than $87 \%$. As authors explain, this kind of system allows patients of rural and remote areas to have regular eye examinations and disease diagnosis. Authors discuss the possibility of extending this system to analyze some other diseases where the diagnosis should be performed through the image analysis, such as skin cancer detection. They also talk about combining the results of these e-Health tools with healthcare systems and AAL proposals.

\subsection{Positioning, localization and detection of obstacles}

The relationships between lifestyle behaviors and health outcomes usually are based on selfreported data. In addition, these data can be very useful for making a database of places where we usually go and for detecting possible obstacles that can damage a person with visual problems. To collect these data, we can use wearable cameras or the smartphone's cameras [50]. Figure 7 shows a typical scene where smartphone's cameras are used to detect obstacles and alert users by acoustic/vibration signals when there are obstacles.

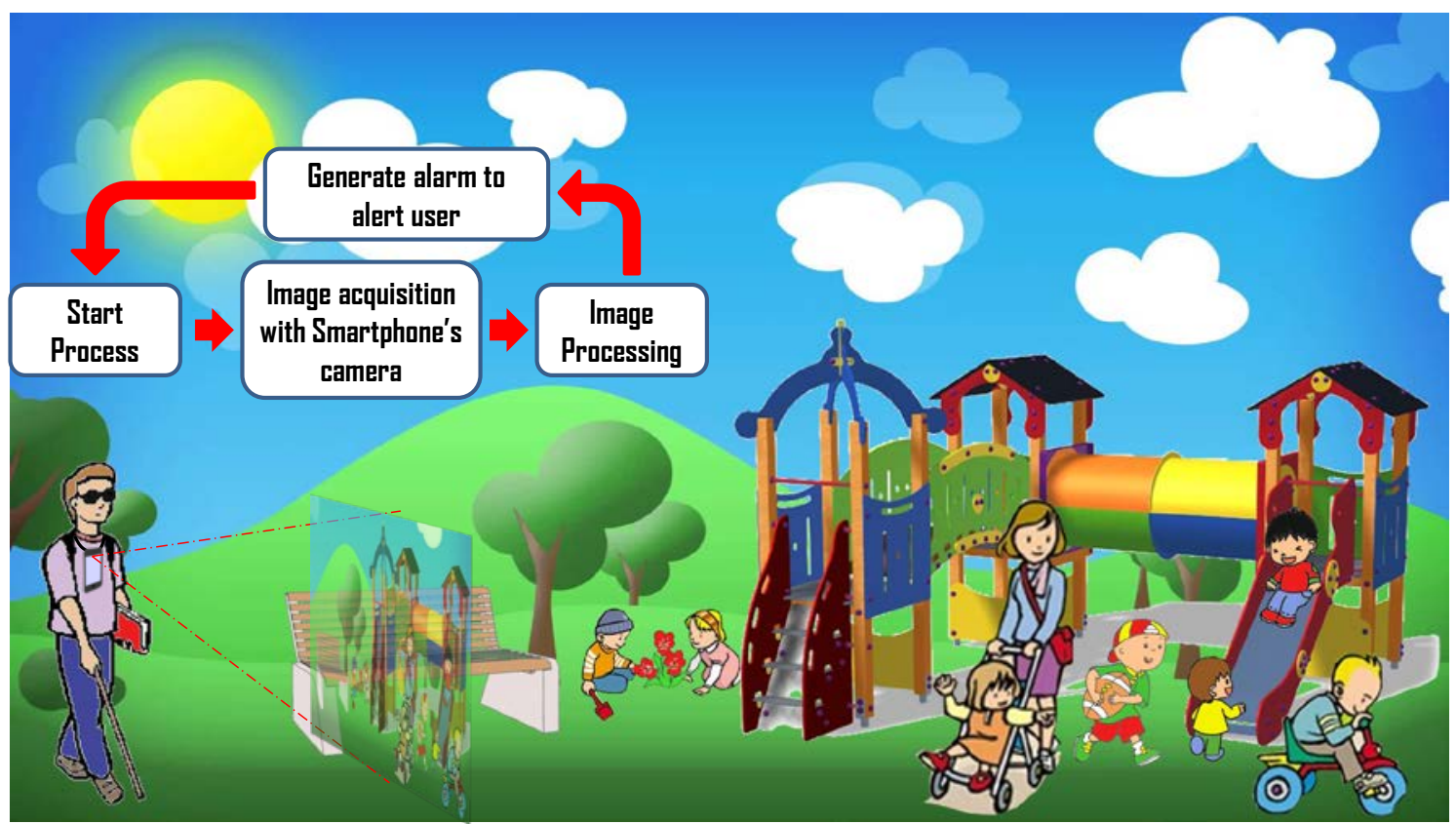

Figure 7. Scene where smartphone's cameras are used to detect obstacles 
Following this hypothesis, D. Kochanov et al. proposed an image guided localization system based on predefined routes with the help of smartphones and image processing techniques [51]. The system is based on the three-dimensional reconstruction images from initial acquired images. The acquired images are sent to the database in order to calculate the location and direction of the camera. The tests of this application show that it is more precise than GPSbased localization systems. Author's proposal is compared with previous research works that presented errors below $3 \mathrm{~m}$ with a probability of 0.75 and below $18 \mathrm{~m}$ with a probability of 0.9 . Authors propose the use of their application for orientation and navigation of visually impaired people, instead of the use of GPS.

In processes of physical rehabilitation, it is important to know the person's mobility profile in a non-clinical setting such as the home or community. For this reason, G. Haché et al. [52] proposed a wearable mobility monitoring system (WMMS) for an objective ubiquitous measurement of mobility. The wearable system is worn as a normal phone (on the person's belt). The prototype of WMMS was created using a smartphone because of its capability, acceptance in the health-care sector and its security. G. Haché et al. selected the BlackBerry smartphone platform for developing the WMMS. The main function of WMMS was the user's mobility state monitoring and to take a photograph when a change of state was detected. After that, these pictures have been used to identify the context of patient mobility events. WMMS performance was evaluated to check its ability for detecting changes of state and the ability to identify context from the pictures. The validation of WMMS demonstrated good potential for people mobility monitoring.

R.Tapu et al. [53] propose a real-time obstacle detection and classification system, based on a smartphone, designed to assist visually impaired people to safely navigate in indoor and outdoor environments. The system is attached to the user with the help of a chest harness. The system analyzes the environment and generates an alarm to alert them about the presence of static/dynamic obstacles few meters around them and to provide assistance for moving in both outdoor and indoor spaces. The application starts selecting several points of interest extracted from an image grid and tracked using the multiscale Lucas - Kanade algorithm. After that, the system background motion is analyzed through homographic transforms. Other objects movements are processed by an agglomerative clustering technique. As a function of the distance to the object, each one is tagged as urgent or normal and a motion vector orientation is created. The result shows that this application is effective for image sequence analysis with high accuracy rates.

\section{Applications and proposals that use the smartphone's Microphone}

The use of smartphone's microphone and headphones can provide great benefits in the field of e-health and AAL. Some papers are related with this issue. We can classify these works based on their applicability. For example, there are studies developed in the field of monitoring to establish diagnosis and in the field of rehabilitation to study sleep disorders or stress episodes. This section is going to show some of the most important applications based on the use of smartphone's microphone and headphones. 


\subsection{Establish diagnoses using audio processing}

Within this area, we can find very interesting proposals as shown below.

Chen et al. [54] proposed a prototype called Listen-to-Nose. It is a phone-based system that can be used to collect reference data, so doctors can diagnose the cause of few symptoms which are related to nasal problems. The Listen-to-Nose is an application that, by means of sound patterns recognition corresponding to blowing or sneezing, can detect nasal symptoms such as a runny nose, stuffy nose, and sneezing. The system can be divided in two parts; the client-side and the server-side (see Figure 9). On the client-side, the application records sounds using the smartphone's microphone and periodically classifies the audio data using an acoustic recognition model. This model classifies sounds as blowing the nose or sneezing, and discards other audio data such as silence and conversations. It also obtains contextual information including location and time. This helps the system to detect when and where the user experiences allergic reactions. On the server-side, the application identifies the audio source (nose blowing, sneezing, or a mistaken event) and potential associated symptoms such as runny or stuffy nose. Data are stored for later use to enhance the accuracy of future events.

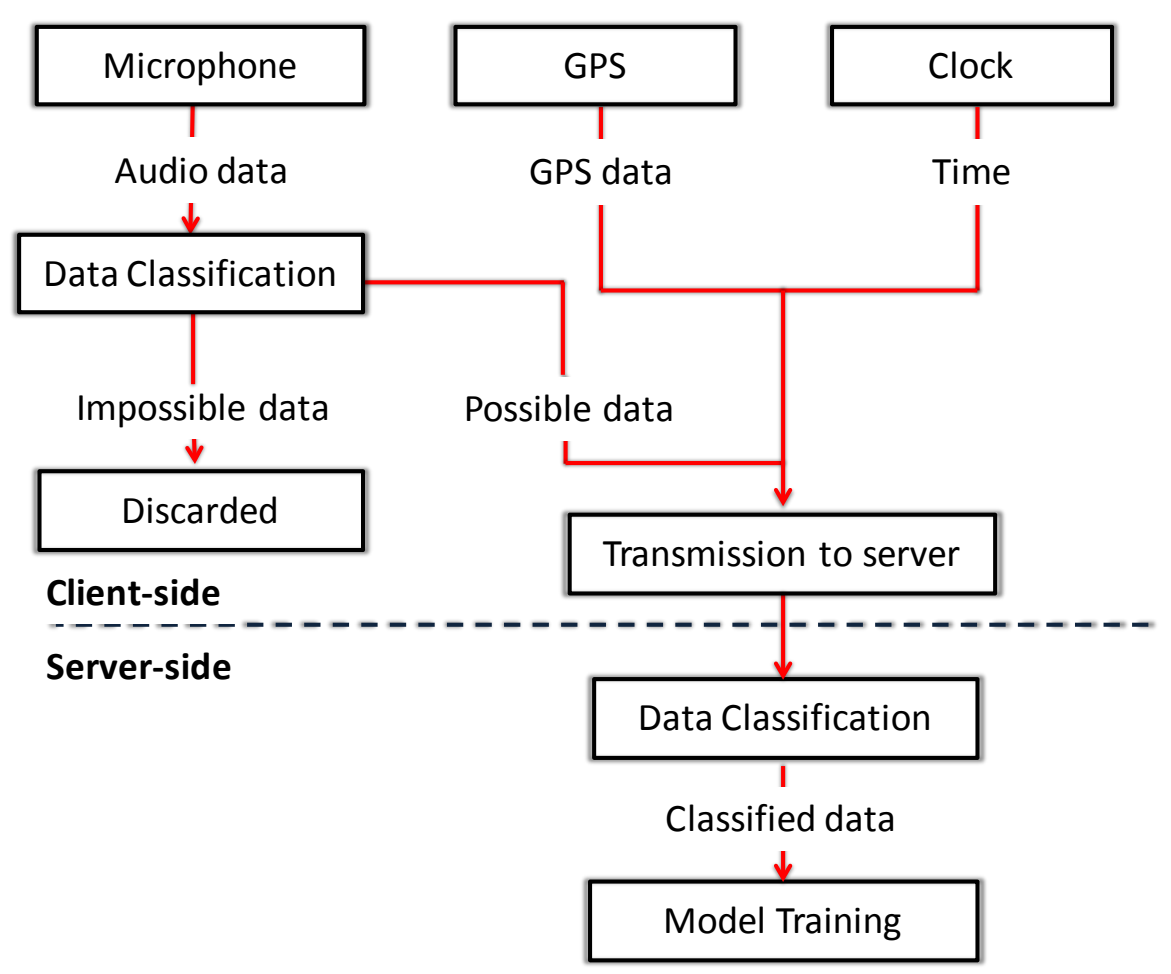

Figure 9. Design of Listen-to-Nose

Alqassim et al. [55] presented the design and implementation of a user-friendly mobile application called Sleep Apnea Monitor (SAM). It has been developed for multiple platforms (Windows and Android). The application is able to monitor and detect symptoms of sleep apnea using the smartphone's built-in sensors. SAM allows users to know whether or not they are likely to have sleep apnea before continuing with more expensive and advanced sleep tests. The application sends to the doctors and sleep specialists patients' records and allows 
them to confirm their initial diagnosis. It uses voice recording software and the mobile built-in accelerometer to record the users breathing patterns and movements during their sleep. In addition, using GSM, the application can determine the patients' location. As Figure 10 shows, the recorded data is securely sent to a remote server using Wi-Fi or 3G connection. On the server side, a statistic analysis of data is performed to assist in diagnosis patients' condition. After that, the results are securely sent back to the users' phone and stored on the server's database as well. The database is accessible via a web server by the doctors with privileged access to users' records. Finally, the system can be configured to send a text message to the user with the outcome. The system was successfully tested following the performance and design requirements. The application and its functionality were verified using 55 samples with very promising results.

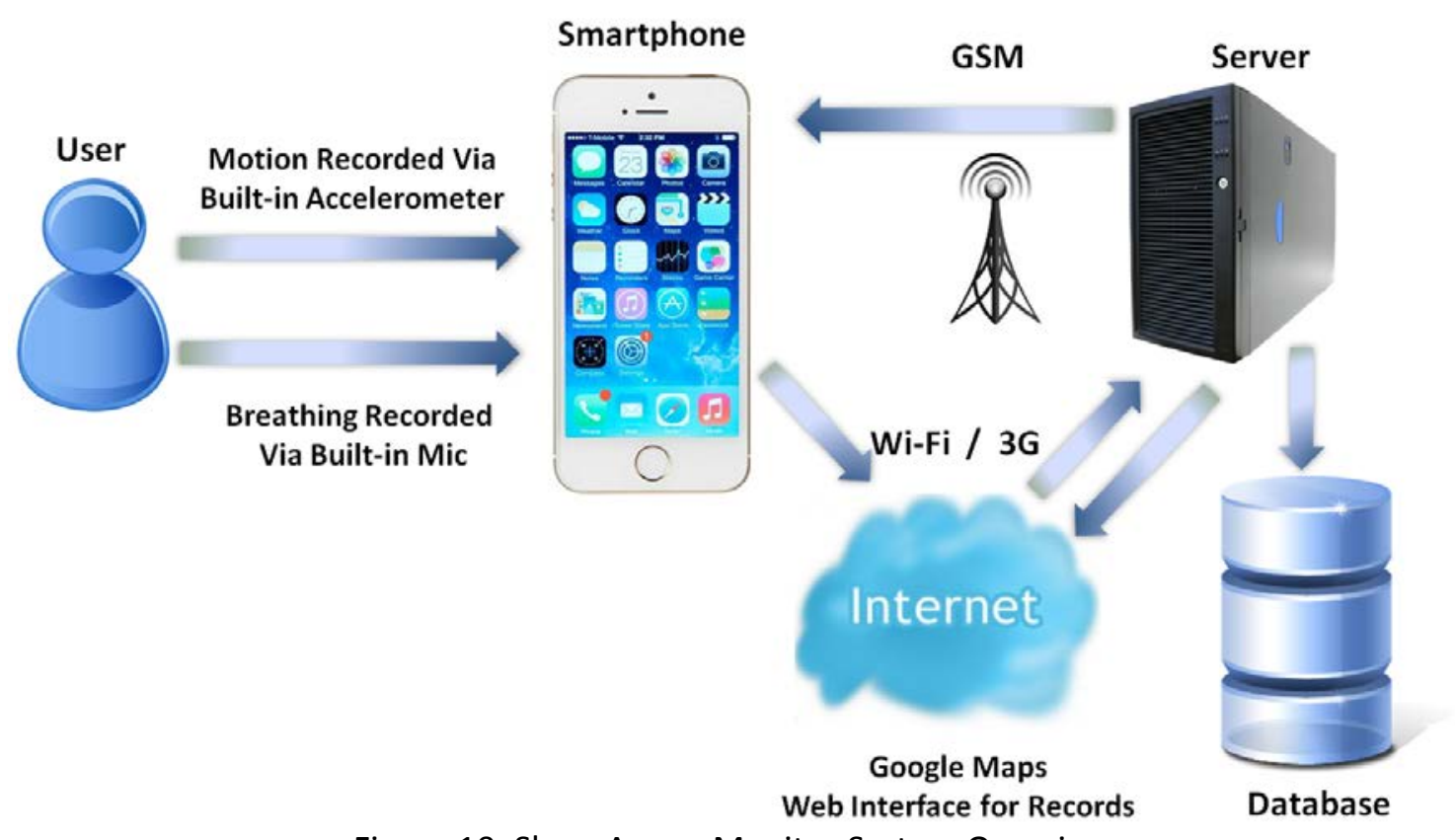

Figure 10. Sleep Apnea Monitor System Overview

Larson et al. [56] present SpiroSmart, a low-cost mobile phone application that performs spirometry sensing using the built-in microphone. The smartphone's microphone records the exhalation. The procedure is as follows. The user holds the smartphone at approximately arm's length, breathe in his/her full lung volume and forcefully exhale at the screen of the phone until the entire lung volume is expelled. Then, the application sends the audio data to a server which calculates the exhaled flow rate by estimating models of the user's vocal tract and the reverberation of sound around the user's head. Flow rate is estimated by calculating the envelope of the sound as a function of time performing resonance tracking in the frequency domain while white noise gain through linear prediction is measured. The authors concluded that SpiroSmart is able to compute and provide flow rates and graphs similar to those generated in home or clinical Spirometers. SpiroSmart is not intended to be a substitute of clinical spirometry, but rather a home-based solution that can increase compliance and monitoring through the convenience of a mobile phone. There are some limitations, for example, inhalation measures are also recorded in clinical spirometry, but inhalation is inaudible and hence it cannot be measured with SpiroSmart. Another limitation is that the 
setting where the test is performed must remain relatively quiet. Finally, there is a decreased accuracy in FVC (total expelled volume during the expiration) compared to other measures.

\subsection{Field of rehabilitation}

Franco et al. [57] propose a smartphone-based audio-biofeedback (ABF) system based on a Kalman Filter that uses the inertial sensors of a Smartphone to estimate $3 D$ angulation of the trunk. The iBalance-ABF system is a smartphone-based ABF balance system that offers to its user a wireless, portable, lightweight and low-cost balance testing and rehabilitation training tool suitable for home use. It also allows the rehabilitation team to quantitatively and objectively assess the patients balance ability. The system is able to show data in real-time to create a customized balance training program by adaptively configuring and adjusting the system BF parameters (e.g., balance exercise difficulty) based on each patient's individual ability/progress/needs/preferences and/or goals, and to track patient's progress history and compliance in order to improve the quality of care. As for existing ABF balance systems, the DeadZone (DZ) is considered as a zone in which a user does not need any extra information to stabilize upright posture. When he/she is swaying outside this $D Z$, the user receives $A B F$ to correct sway to within the $\mathrm{DZ}$ in order to stabilize upright posture.

\subsection{Applications to study the stress}

An interesting application is the proposal presented by Lu et al. [58]. They presented a system called StressSense. It is a method for detecting the stress using smartphone microphones and adapting universal models of stress to specific individuals or scenarios using Maximum a Posteriori (MAP) adaptation scheme. StressSense achieves $81 \%$ and $76 \%$ accuracy for indoor and outdoor environments, respectively. StressSense can be implemented for Android phones and runs in real-time. Unlike physiological sensors, microphones do not require contact with the human body and are compatible with all mobile phones. Using a non-iterative MAP adaptation scheme for Gaussian mixture models, authors demonstrated that it is feasible to customize a universal stress model to different users and different scenarios using only few new data observations with low computational overhead. Authors conducted an initial study of cognitive load related stress in job interviews and outdoor job execution tasks. For neutral voice, they used reading data. Then, they investigated three training schemes to model stress: universal model, personalized model and speaker adapted universal model. To evaluate StressSense, authors conduct three experiments using the dataset and stress models. First, they evaluated the importance and effectiveness of different vocal features for different acoustic environments. Second, they studied the success of stress models trained and tested in specific scenarios, i.e., either fully indoors or fully outdoors. Finally, authors investigated the stress classification performance under mixed acoustic environments. Each one of the StressSense classification models evaluated in the paper presents tradeoffs in terms of training and the burden of the users. Authors plan to enhance StressSense application using an adaptive pipeline that uses the self-train model for speaker adaption and the supervised adaption model for environment adaption. 


\section{Commercial Android applications based on Smartphone's camera and microphone for AAL and e-health}

There are several Android Applications for smartphone available in Google Play Store that use the smartphone's camera and/or microphone as a tool for AAL and healthcare. The most relevant examples will be cited in this section. We have tried to choose different types of Apps in order to show the wide range of possibilities. We only cite one App for each parameter. A review of healthcare App was done in [59], but this study was not focused only in AAL and ehealth applications based on a determined sensor. Our study is focused only in multimedia applications that uniquely use the camera and/or microphone.

\subsection{App that uses the camera:}

MedCam [60]: This App is able to measure the breathing and the pulse rate of a user using the smartphone's camera. It uses the frontal camera. The screen shows the images taken by the camera and two rectangles. The user must adjust the distance and position of the smartphone in order to march the upper rectangle with his/her face and the lower rectangle with his/her chest. Then, pushing the start button, the record starts. After few seconds, the screen shows the breathing and the pulse rate. For measuring the pulse, the App uses the camera to see the small changes of the reflectivity in the face of user (each heart beat changes the reflectivity of the face in 1\%). For measuring the breathingthe App analyzes the movement of the user's chest. The App has a size of $941 \mathrm{kBthe}$. Current version is 1.12 and it has between 10,000 and 50,000 downloads. The average score is 3.5 with 79 votes. It is included in the category of Health \& Fitness and it is free.

Color Detector [61]: This App uses the smartphone's camera to recognize the predominant color in the captured image. This App is especially interesting for colorblind people or other eyes disorders. Once the App is running, the camera image appears in the screen with a circle that indicates the area of color to be guessed. After taking the photo, the screen shows the name of the colors using words and the RGB values. The color is determined according to the main RGB values of the pixels in the area. The App has a size of $68 \mathrm{kB}$ and the current version is 1.9.It has between 50,000 and 100,000 downloads with an average score of 3.6 with 526 votes. It is included in the category of Tools and it is free.

Colorblind Assistant [62]: This App uses the smartphone's camera to recognize the 5 main colors of a photo. This App is especially interesting for colorblind people and other eyes disorders. When the App runs, the image camera appears in the screen and shows the name of colors according to the RGB values. The App has a size of $63 \mathrm{kB}$. The current version is 2.0 and it has between 500 and 1,000 downloads. The average score is 2.7 with 3 votes. It is included in the category of Health \& Fitness and it is free.

The volCe for Android [63]: This App uses the smartphone's camera to convert the images into sounds offering augmented reality for blind. It uses treble or bass tones to indicate the height of the captured object. With high or low volume indicates the clarity of it. The App reads the image from left to right each second. Clear areas are identified by a short beep, clear 
square solids by a sonic boom, and vertical grid as a rhythm. The App is compatible with stereo headphones. It also includes a color detector, a GPS and a compass that tells in loud the location and orientation. The App has a size of $1.6 \mathrm{MB}$, the current version is $1.84 \mathrm{~b}$ and it has between 100,000 and 500,000 downloads with an average score of 3.5. It has received 76,526 votes. It is included in the category of Health \& Fitness and it is free.

Voice Cam for Blind [64]: This App uses the smartphone's camera to read in loud the English words detected in a picture. The App has a voice guidance that helps to known the relative position of the text to the camera. This guidance can be activated or deactivated with a button. The number of words detected and the number or rows can be known pressing a button. The App does not need Internet connection when it is running. The App has a size of 7.0MB and the current version is 2.01 .It has between 10 and 50 downloads. Its average score is 3.7 with 3 votes. It is included in the category of Health \& Fitness and it costs $0.70 €$.

Cardiograph [65]: This App uses the smartphone's camera and the flash to detect the heart rate. The App relates the changes in the skin color with the heartbeat, using the flash for illuminating the finger. The measure takes 10 s and the heartbeats can be seen in the screen by a graphic. Users can select the activity that he/she is doing in order to record the data in a profile. Different profiles can be maintained in just one smartphone. This App can be very useful for people with heart diseases or anxiety, or even for people with medication that could produce arrhythmias. The size of App and its version varies depending on the smartphone. It has between 10,000,000 and 50,000,000 downloads and an average score of 3.8 with 129,798 votes. It is included in the category of Health \& Fitness and it is free.

\subsection{App that uses the microphone}

MyEarDroid [66]: This App uses the smartphone's microphone to detect sounds and alert the users about them. It helps people with hearing impairment. It can distinguish between different types of typical sounds in home environments. The App alerts the user with vibration and visual notifications about the detected sound. It compares the sound with a database of sounds, which includes doorbell, telephone, intercom, alarm clock and alarms. The App offers the possibility of recording his/her own sounds of doorbell, telephone, etc. to improve the results and also incorporate them to the database. The App has a size of $3.8 \mathrm{MB}$. The current version is 1.0.2 and it has between 1,000 and 5,000 downloads with an average score of 3.5 with 33 votes. It is included in the category of Health \& Fitness and it is free.

Quit Snoring [67]: This App uses the smartphone's microphone to detect snoring sounds during the user is sleeping. It does not help to completely stop the snoring but it can help to stop individual snoring episodes. The App shows the user how often and how loud he/she snores thanks to the records of the sleep sessions. In the morning, a summary is shown in the screen with the number of times the user snores and how loud the snoring was. It can also be visualized with a graph. The App offers the possibility of delivering a small "nudge" when it detects a snoring episode. This "nudge" is aimed to stop the snoring episode. The "nudge" can be a vibration or an alarm sound. Users can choose which sound should be used from a database or records their own sound. The snoring episodes can be heard after the sleep 
session. The App has a size of 6.3MB and the current version is 1.0.15. It has between 100 and 500 downloads with an average score of 2.8 and 8 votes. It is included in the category of Health \& Fitness and it costs $1.46 €$.

SnoreClock [68]: This App uses the microphone to record the sleeping session in order to know if there are snoring episodes or some sounds generated during the sleeping sessions. It automatically stops when the battery level is low. This App can be helpful for people with respiratory problems or sleeping apnea. The App has a size of $2.1 \mathrm{MB}$, the current version is 1.9.5 and it has between 100,000 and 500,000 downloads. The average score is 3.8 with 1995 votes. It is included in the category of Health \& Fitness and it is free.

Breath Counter [69]: This App uses the smartphone's microphone to analyze the breath rate of the user. When the App runs, it requests the user to breath normally near to his/her smartphone during 30 seconds. Instantaneously, the number of breaths per minute is shown in the screen. The user can save and share the data via Facebook, Stream or Gmail, among others. The App also provides the typical values of breaths per minute at different ages. The App has a size of 2.2MB. It has between 1,000 and 5,000 downloads and an average score of 3.5 with 10 votes. It is included in the category of Health \& Fitness and it is free.

\section{Discussion}

This section compares the works reviewed in the previous sections. We compare them in terms of device used, kind of application and the number of application that uses each device as well as the evolution of each technology. Finally, we present a brief discussion about the different kind of commercial applications we have found for android OS.

First of all, it is interesting to show the elements used for developing the analyzed applications. Figure 17 shows that the element most used to develop applications for AAL or e-Health purposes is the smartphone's camera. It is used in the $67 \%$ of applications and the smartphone's microphone in used in the $33 \%$ of applications.

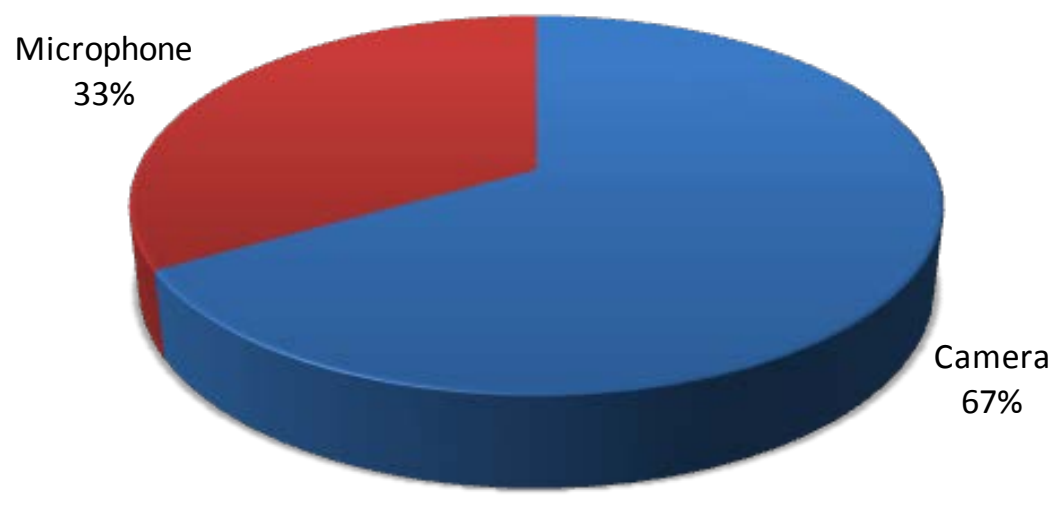


Figure 17. Elements used for implementing different applications for AAL and e-Health applications.

The number of papers published using the audio of video captured by a smartphones for AAL or e-health applications along the years is shown in Figure 18. The first contribution appeared in 2010 and it used the smartphone's earphones. The number of papers per year has increased until 2012. However, the total number of contributions decreased in 2013. By contrast, the number of contributions that uses the smartphone's camera still increased.

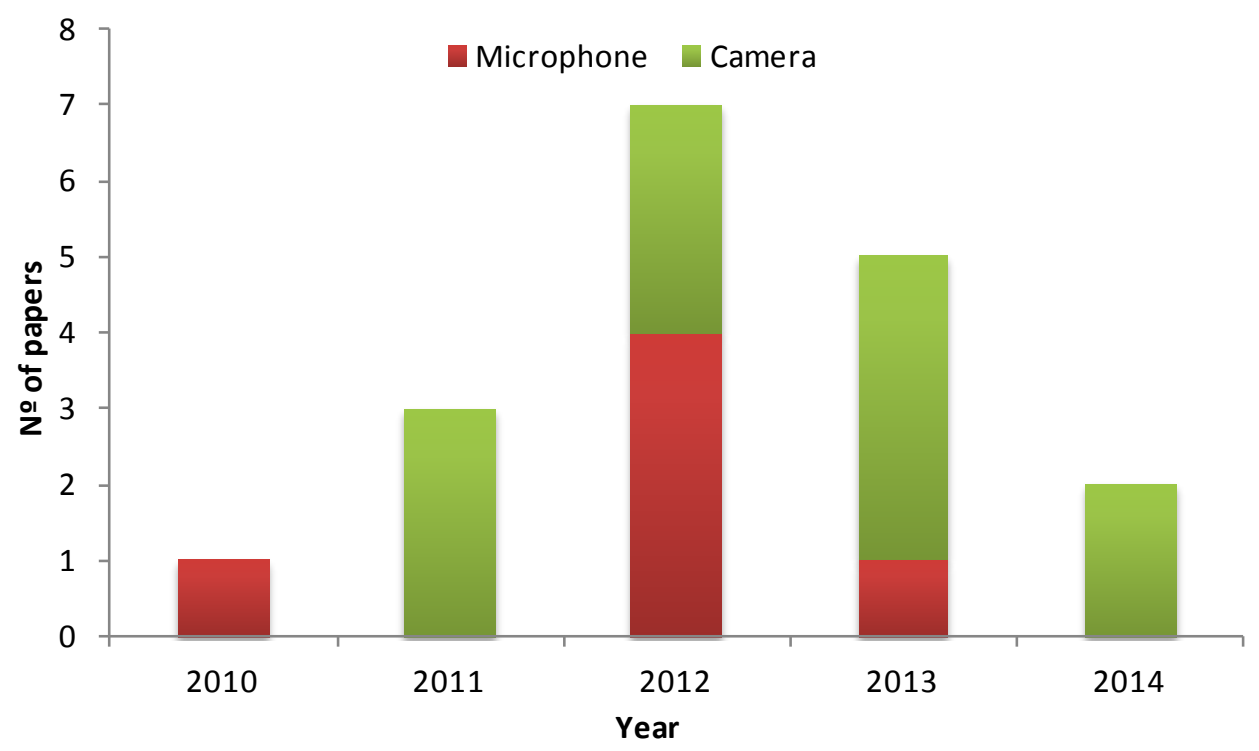

Figure 18. Variation of contributions per year that uses the audio and video of smartphones for AAL and e-Health purposes.

Figure 19 presents the main purposes for AAL and e-Health applications that use the smartphone's camera. The most relevant purpose is aimed to people monitoring ( $45 \%$ of the applications). The diagnosis is the second application most used (33\%), followed by mood recognition and rehabilitation training (11\%, each one). Figure 20 presents the main purposes for AAL and e-Health applications that use the smartphone's microphone or earphone. Again, the main purpose is the users monitoring, with a percentage of $54 \%$. It is followed by diagnostic purposes (31\%) and the mood recognition and rehabilitation training tools are only used in the $7 \%$ and $8 \%$ of cases, respectively. 


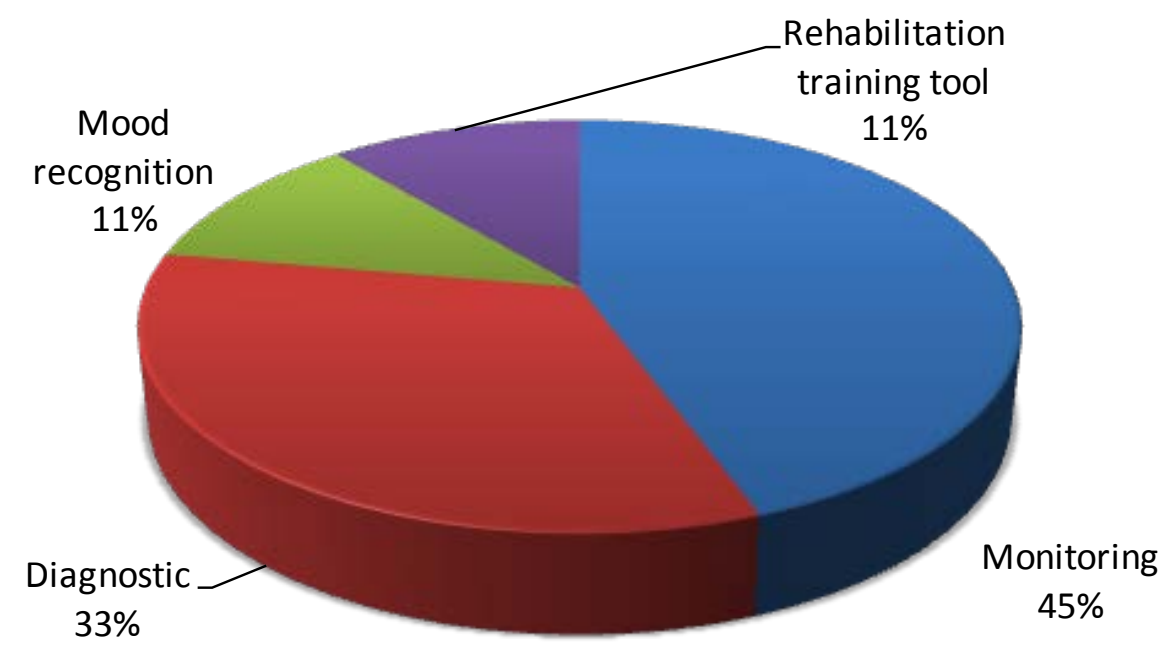

Figure 19. Main purposes for AAL and e-health systems based on smartphone's camera

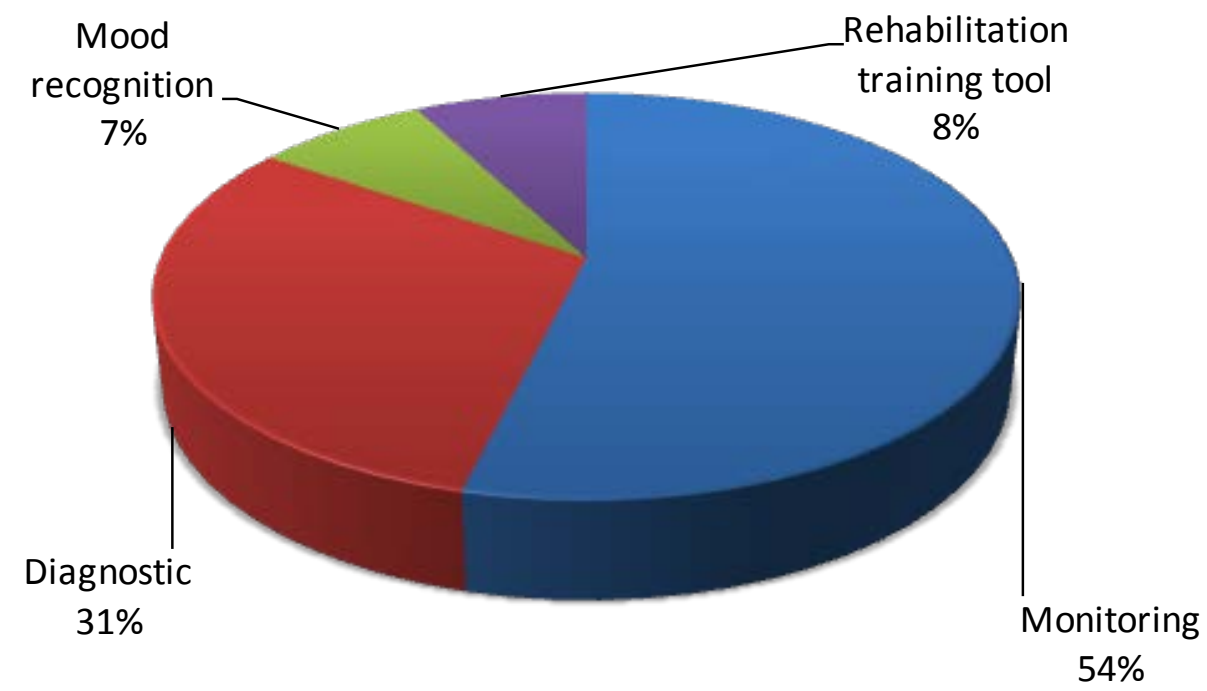

Figure 20. Main purposes for AAL and e-Health systems based on smartphone's microphone and earphones

We can also analyze the parameters that each application is trying to measure. Figure 21 shows the purposes when smartphone's cameras are used. The hearth beat is the most measured parameter ( $42 \%)$, the diseases recognition is also an important topic. The $25 \%$ of these applications are specialized on skin diseases and $8 \%$ of them on retina diseases. Object recognition $(8 \%)$ and the location $(17 \%)$ are also present among these video-based applications. The same analysis is performed for audio-based applications. Results are shown in Figure 22. The nose-related diseases is the most developed topic with a 37\%. The movement of users is the second application most predominant over other applications (25\%). The breath rate is as important as the spirometry with a percentage of usage of $13 \%$ and finally, heart rate is the lowest one with $12 \%$. 


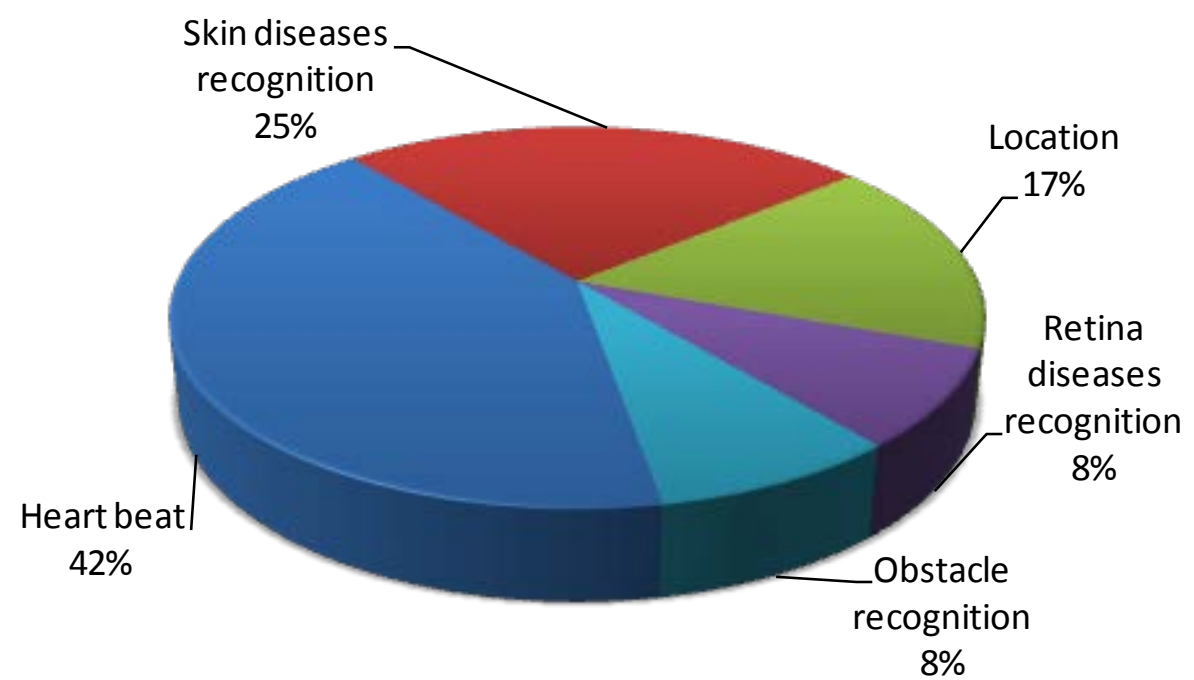

Figure 21. Parameters measured by applications based on smartphone's cameras

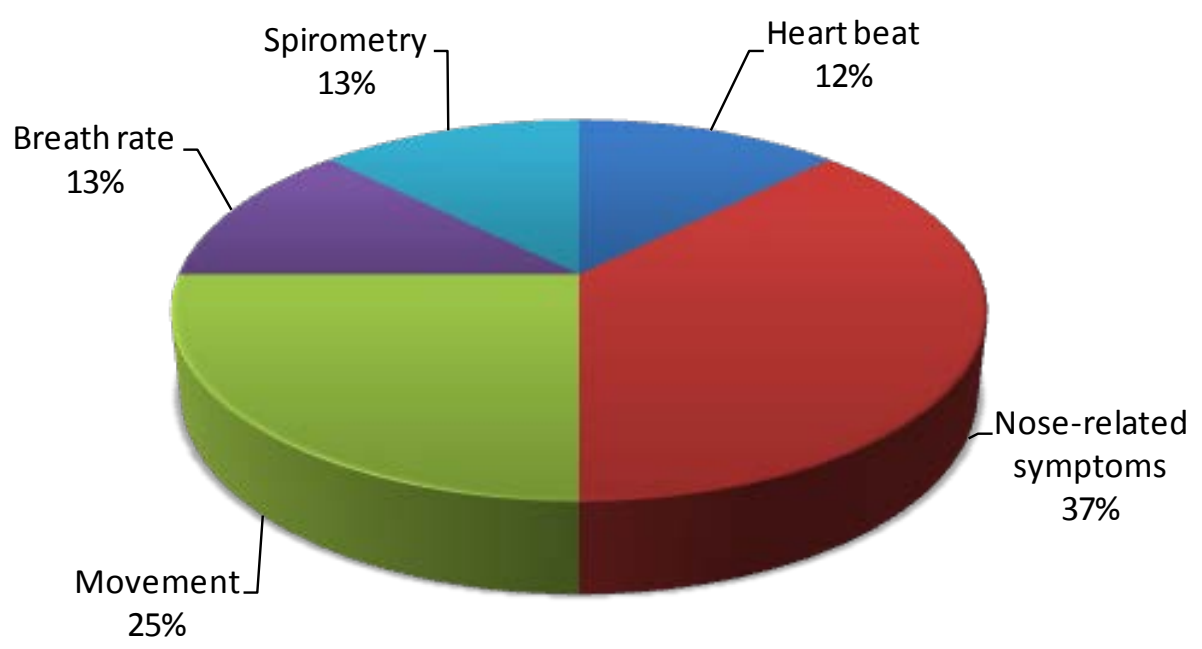

Figure 22. Deployment purpose based on smartphone's microphones and earphones

Table I and II present a classification of all reviewed works in Section 2 and Section 3. Table I shows the papers classified according to the used sensor and the purpose of the application such as monitoring, diagnostic or mood recognition, among others. Most of applications are developed for monitoring purposes. Table II shows these works classified according to the purpose of the application such as heart beat, breathing rate, skin diseases recognition, etc. The purpose with most developments is the hearth beat followed by nose diseases. Obstacles recognition and spirometry are the less developed ones.

Table I. Classification according to the function and used sensor

\begin{tabular}{|c|c|c|c|c|c|c|}
\hline \multirow{2}{*}{ Ref. } & \multirow{2}{*}{ Name/ brief description } & \multirow{2}{*}{ Sensor } & \multicolumn{4}{|c|}{ Function } \\
\hline & & & Monitoring & Diagnostic & Rehabilitation & $\begin{array}{c}\text { Mood } \\
\text { recognition }\end{array}$ \\
\hline
\end{tabular}




\begin{tabular}{|c|c|c|c|c|c|c|}
\hline [54] & Listen-to-Nose & MP & $\checkmark$ & $\checkmark$ & & \\
\hline [55] & Sleep Apnea Monitor & MP & $\checkmark$ & $\checkmark$ & & \\
\hline [56] & SpiroSmart & MP & $\checkmark$ & & & \\
\hline [57] & iBalance-ABF & MP & & & $\checkmark$ & \\
\hline [58] & StressSense & MP & & & & $\checkmark$ \\
\hline [41] & Atrial fibrillation detection & $\mathrm{CM}$ & $\checkmark$ & & & \\
\hline [42] & Application to measure pulse in real-time & $\mathrm{CM}$ & $\checkmark$ & & & \\
\hline [43] & Provision of cardiac psychophysiological data & $\mathrm{CM}$ & $\checkmark$ & & & \\
\hline [52] & Wearable Mobility Monitoring System & $\mathrm{CM}$ & $\checkmark$ & & & \\
\hline [53] & A real-time obstacle detection and classification & $\mathrm{CM}$ & $\checkmark$ & & & \\
\hline [51] & Image guided localization based on predefined routes & $\mathrm{CM}$ & $\checkmark$ & & & \\
\hline [44] & Smartphone Pulse Rate Variability & $\mathrm{CM}$ & $\checkmark$ & & & \\
\hline [45] & Android application to measure Heart Rate & $\mathrm{CM}$ & $\checkmark$ & & & \\
\hline [46] & Recognition of possibles melanomas & $\mathrm{CM}$ & & $\checkmark$ & & \\
\hline [47] & $\begin{array}{l}\text { Intelligent system to analyze skin images to identify } \\
\text { cancer }\end{array}$ & $\mathrm{CM}$ & & $\checkmark$ & & \\
\hline [48] & $\begin{array}{l}\text { 7-Point Checklist for Melanoma Detection on Smart } \\
\text { Devices }\end{array}$ & $\mathrm{CM}$ & & $\checkmark$ & & \\
\hline [49] & Android platform for retinal disease diagnostic & $\mathrm{CM}$ & & $\checkmark$ & & \\
\hline
\end{tabular}

Earphones (EP), Micropones (MP) and Camera (CM)

Table II. Classification according to the sensed parameter

\begin{tabular}{|c|c|c|c|c|c|c|c|c|c|c|}
\hline \multirow[b]{2}{*}{ Ref. } & \multirow[b]{2}{*}{ Year } & \multicolumn{9}{|c|}{ Sensed parameter } \\
\hline & & $\begin{array}{c}\text { Heart } \\
\text { beat }\end{array}$ & $\begin{array}{l}\text { Nose-related } \\
\text { symptoms }\end{array}$ & $\begin{array}{c}\text { Skin } \\
\text { diseases }\end{array}$ & Movement & Location & $\begin{array}{c}\text { Retina } \\
\text { diseases }\end{array}$ & $\begin{array}{l}\text { Breath } \\
\text { rate }\end{array}$ & $\begin{array}{c}\text { Obstacle } \\
\text { recognition }\end{array}$ & Spirometry \\
\hline$[42]$ & 2011 & $\checkmark$ & & & & & & & & \\
\hline [48] & 2011 & & & $\checkmark$ & & & & & & \\
\hline [52] & 2011 & & & & & $\checkmark$ & & & & \\
\hline [41] & 2012 & $\checkmark$ & & & & & & & & \\
\hline [44] & 2012 & & & & & & & $\sqrt{ }$ & & \\
\hline$[46]$ & 2012 & & & $\checkmark$ & & & & & & \\
\hline [55] & 2012 & & $\checkmark$ & & & & & & & \\
\hline [56] & 2012 & & $\checkmark$ & & & & & & & \\
\hline [54] & 2012 & & $\checkmark$ & & & & & & & \\
\hline [58] & 2012 & & & & & & & & & $\checkmark$ \\
\hline [59] & 2012 & & & & $\checkmark$ & & & & & \\
\hline [43] & 2013 & & & & & & & & & \\
\hline [53] & 2013 & & & & & $\checkmark$ & & & & \\
\hline [57] & 2013 & & & & & & & & $\checkmark$ & \\
\hline [45] & 2013 & $\checkmark$ & & & & & & & & \\
\hline$[47]$ & 2013 & & & $\checkmark$ & & & & & & \\
\hline [50] & 2013 & & & & & & $\checkmark$ & & & \\
\hline [49] & 2014 & & & $\checkmark$ & & & & & & \\
\hline [51] & 2014 & & & & & & $\checkmark$ & & & \\
\hline
\end{tabular}


Finally, in regard to commercial application, we found a total of 10 different kinds of applications for AAL or e-Health purposes that uses the embedded camera and/or microphone in smartphones. Most of these Apps are camera-based (6 out of 10 with a percentage of $60 \%$ ) and only 4 of them are microphone-based (40\%). Their main purposes are shown in Figure 23. As we can see, most of these applications are designed to help blinds and for monitoring different parameters such as pulse, breath rate and snoring. Regarding to the price, the $80 \%$ of the analyzed applications are free. Only 2 of them are paid and their price does not reach $1.50 €$.

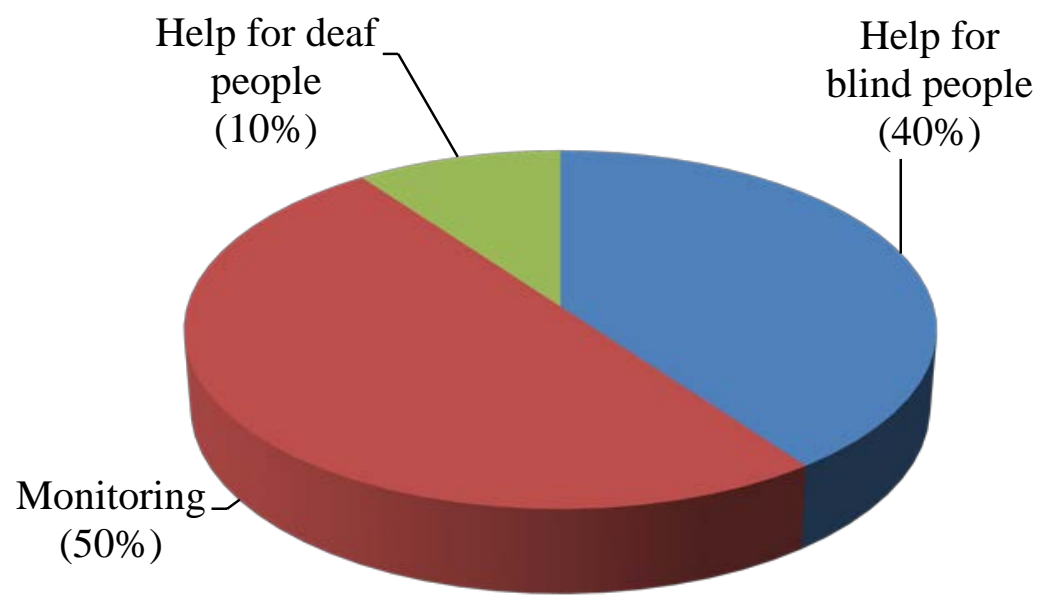

Figure 23. Main purposes of Android App for AAL and healthcare that uses camera and/or microphone

When research works are compared with the real Apps, it is possible to see some similitudes. The percentage of research works based on the camera use is $67 \%$ while the real applications based on the camera are $60 \%$. In both cases, most of these applications (real Apps or research works) are based on the use of camera. As in proposals, in real mobile applications the main purpose is monitoring. The percentage of real App for monitoring is $50 \%$ where the $45 \%$ of them use the camera and $54 \%$ of them use the microphone and/or earphones. However, proposals for diagnostic establishment, mood recognition of rehabilitation have not yet been developed in real applications. Moreover, there are a lot of real applications focused on increasing the quality of life of blind and deaf people, but in the review of research papers we do not find any reference to that kind of purpose.

\section{Conclusions}

The ageing of population is a reality in many developed countries and many problems are related to this fact. One of them is related to the special needs of these people. Too many elderly people may cause that the current healthcare systems will be not sustainable. The AAL systems and e-Health applications are possible solutions for these problems. 
In this area, several mobile monitoring systems have been developed using different technologies. The embedded sensors in mobile devices and smartphones can be used to bring new solutions and applications to AAL scenarios.

Multimedia data are useful for different e-Health applications and AAL systems and platforms. This paper has presented a survey on applications that use multimedia data from smartphones for AAL and e-Health purposes. We did not find any previous work that analyzed this information. Firstly, we presented several works where authors proposed AAL or e-Health applications based on the smartphone's camera. We grouped these applications according to their different topics as hearth beat monitoring or skin diseases recognition, among others. The same study was performed for AAL or e-Health applications based on the smartphone's microphone and earphone. We also presented a comparison with the data collected. Some analyses were discussed such as number of contributions per year and the most used elements (camera, earphones or microphones). We also presented several current commercial applications for Android smartphones that can be found in Google Play Store.

When we performed this work, we observed that almost all published works used the microphone or the camera, but not the combination of both (we found just one that used the video and audio).

Our future work will be focused on developing systems that use both, the microphone and camera. These systems may be more complete because of the use of both multimedia sensors. Moreover, the fact of having several types of sensors measuring the different types of parameters will allow us to build different sensor networks with different purposes [70]. Furthermore, as this field is growing hugely, very soon the research community will most probably need a new survey with the works published in next few years.

\section{References}

[1] O'Grady, M. J., Muldoon, C., Dragone, M., Tynan, R., and O'Hare, G. M. (2010). Towards evolutionary ambient assisted living systems. Journal of Ambient Intelligence and Humanized Computing, 1(1): 15-29.

[2] Anderson, G., and Horvath, J. (2004). The growing burden of chronic disease in America. Public health reports, 119(3): 263-270.

[3] Aquilano, M., Cavallo, F., Bonaccorsi, M., Esposito, R., Rovini, E., Filippi, M., and Carrozza, M. C. (2012). Ambient assisted living and ageing: Preliminary results of RITA project. In proceedings of 34th Annual International Conference of the IEEE Engineering in Medicine and Biology Society (EMBC 2012), Aug. 28-Sept. 1, 2012. San Diego USA. (pp. 5823-5826)

[4] Storf, H., Kleinberger, T., Becker, M., Schmitt, M., Bomarius, F., and Prueckner, S. (2009). An event-driven approach to activity recognition in ambient assisted living, Lecture Notes in Computer Science, 5859(): 123-132.

[5] Kurniawan, S. (2008). Older people and mobile phones: A multi-method investigation. International Journal of Human-Computer Studies, 66(12): 889-901. 
[6] Furqan Hameed Khan, Zeashan Hameed Khan, (2010). A Systematic Approach for Developing Mobile Information System based on Location Based Services, Network Protocols and Algorithms, 2(2): 54-65

[7] Al-Attas, R., Yassine, A., and Shirmohammadi, S. (2012). Tele-Medical Applications in Home-Based Health Care. In proceeding of the 2012 IEEE International Conference on Multimedia and Expo Workshops (ICMEW 2012). Jul. 9-13, 2012. Melbourne, Australia. (pp. 441-446).

[8] Weider Yu, Xiao Su, Jason Hansen, (2012). A Smartphone Design Approach to User Communication Interface for Administering Storage System Network, Network Protocols and Algorithms, 4(4): 126-155

[9] Macias, E., Suarez, A., and Lloret, J. (2013). Mobile sensing systems. Sensors, 13(12): 17292-17321.

[10]Sendra, S., Granell, E., Lloret, J., and Rodrigues, J. J. P.C. (2014). Smart collaborative mobile system for taking care of disabled and elderly people. Mobile Networks and Applications, 19(3): 287-302.

[11]Kanjo, E. (2012). Tools and architectural support for mobile phones based crowd control systems. Network Protocols and Algorithms, 4(3): 4-14.

[12]Zhang, D., Xiong, H., Yang, L., \& Gauither, V. (2013). NextCell: predicting location using social interplay from cell phone traces. EEE Transactions on Computers, 64(2): 452-463

[13]Zhang, D., Vasilakos, A. V., \& Xiong, H. (2012). Predicting location using mobile phone calls. ACM SIGCOMM Computer Communication Review, 42(4), 295-296.

[14]Xiong, H., Zhang, D., Zhang, D., \& Gauthier, V. (2012, September). Predicting mobile phone user locations by exploiting collective behavioral patterns. In proceedings of the 9th International Conference on Ubiquitous Intelligence \& Computing and 9th International Conference on Autonomic \& Trusted Computing (UIC/ATC). 4-7 Sept. 2012. Fukuoka, Japan. (pp. 164-171).

[15]Boulos, M. N., Wheeler, S., Tavares, C., and Jones, R. (2011). How smartphones are changing the face of mobile and participatory healthcare: an overview, with example from eCAALYX. Biomedical engineering online, 10(1): 24.

[16]Tudzarov, A., and Janevski, T. (2011). Protocols and algorithms for the next generation 5G mobile systems. Network Protocols and Algorithms, 3(1): 94-114.

[17]Lacuesta, R., Lloret, J., Sendra, S., and Peñalver, L. (2014). Spontaneous Ad Hoc Mobile Cloud Computing Network. The Scientific World Journal, 2014: 1-19.

[18]Xu, X., Shu, L., Guizani, M., Liu, M., and Lu, J. (2014) A Survey on Energy Harvesting and Integrated Data Sharing in Wireless Body Area Networks, International Journal of Distributed Sensor Networks. Article ID 438695

[19]Estepa, A. J., Estepa, R., Vozmediano, J., and Carrillo, P. (2014). Dynamic VolP codec selection on smartphones. Network Protocols and Algorithms, 6(2): 22-37. 
[20]Illiger, K., Hupka, M., von Jan, U., Wichelhaus, D., and Albrecht, U. V. (2014). Mobile Technologies: Expectancy, Usage, and Acceptance of Clinical Staff and Patients at a University Medical Center. JMIR mHealth and uHealth, 2(4): e42.

[21]Monteiro, D. M., Rodrigues, J. J., Lloret, J., and Sendra, S. (2014). A hybrid NFC-Bluetooth secure protocol for Credit Transfer among mobile phones.Security and Communication Networks, 7(2): 325-337.

[22]Rahman, M. A., Hossain, M. S., El Saddik, A. (2013). Context-aware multimedia services modeling: an e-Health perspective. Multimedia Tools and Applications, 73(3): 1147-1176.

[23] Bellini, P., Bruno, I., Cenni, D., Fuzier, A., Nesi, P., Paolucci, M. (2012). Mobile Medicine: semantic computing management for health care applications on desktop and mobile devices. Multimedia Tools and Applications, 58(1): 41-79.

[24]Dale, O., Solheim, I., Halbach, T., Schulz, T., Spiru, L., and Turcu, I. (2013). What seniors want in a mobile Help-On-Demand service. In proceedings of the Fifth International Conference on eHealth, Telemedicine, and Social Medicine (eTELEMED 2013). Feb. 24 Mar. 1, 2013. Nice, France. (pp. 96-101).

[25]Hoseini-Tabatabaei, S. A., Gluhak, A., and Tafazolli, R. (2013). A survey on smartphonebased systems for opportunistic user context recognition. ACM Computing Surveys (CSUR), 45(3): 1-51. Paper No. 27.

[26]Kawano, Y., and Yanai, K. (2014). FoodCam: A real-time food recognition system on a smartphone. Multimedia Tools and Applications,Published online:April 2014: 1-25.

[27]Macías, E., Abdelfatah, H., Suárez, A., and Cánovas, A. (2011). Full geo-localized mobile video in Android mobile telephones. Network Protocols and Algorithms, 3(1): 64-81.

[28]Macias, E., Lloret, J., Suarez, A., and Garcia, M. (2012). Architecture and protocol of a semantic system designed for video tagging with sensor data in mobile devices. Sensors, 12(2): 2062-2087.

[29]Alemdar, H., and Ersoy, C. (2010). Wireless sensor networks for healthcare: A survey. Computer Networks, 54(15): 2688-2710.

[30]Acampora, G., Cook, D. J., Rashidi, P., and Vasilakos, A. V. (2013). A survey on ambient intelligence in healthcare. Proceedings of the IEEE, 101(12): 2470-2494.

[31]Falk, T. H., and Maier, M. (2013). Context awareness in WBANs: a survey on medical and non-medical applications. IEEE Wireless Communications, 20(4): 30-37.

[32]Chiarini, G., Ray, P., Akter, S., Masella, C., and Ganz, A. (2013). mHealth technologies for chronic diseases and elders: A systematic review. IEEE Journal on Selected Areas in Communications,31(9): 6-18.

[33]Su, X., Tong, H., and Ji, P. (2014). Activity recognition with smartphone sensors. Tsinghua Science and Technology, 19(3): 235-249.

[34]Chaaraoui, A. A., Climent-Pérez, P., and Flórez-Revuelta, F. (2012). A review on vision techniques applied to human behaviour analysis for ambient-assisted living. Expert Systems with Applications, 39(12): 10873-10888. 
[35]Poppe, R. (2010). A survey on vision-based human action recognition. Image and vision computing, 28(6), 976-990.

[36]Smith, A. (2013) Smartphone Ownership 2013. On-line Report June 5, 2013. In Pew Research Center's Internet \& American Life Project website. Available at: http://www.pewinternet.org/2013/06/05/smartphone-ownership-2013/ [Last access: Nov.25,2014]

[37]García, M., Lloret, J., Bellver, I., and Tomás, J. (2013) Intelligent IPTV Distribution for Smart Phones (Book Chapter 13). In Intelligent Multimedia Technologies for Networking Applications. IGI Global

[38]Smartphone Milestone: Half of Mobile Subscribers Ages 55+ Own Smartphones Mobile. Online report.(April 22,2014). In the Nielsen Company website. Available at: http://www.nielsen.com/us/en/insights/news/2014/smartphone-milestone-half-ofamericans-ages-55-own-smartphones.html [Last Access: Nov 25,2014]

[39]M.W. Brault, (2010). Americans With Disabilities: 2010. Household Economic Studies. In United States Census Bureau website. Available at: www.census.gov/prod/2012pubs/p70131.pdf [Last access: December 16, 2014]

[40]Cardinaux, F., Bhowmik, D., Abhayaratne, C., and Hawley, M. S. (2011). Video based technology for ambient assisted living: A review of the literature. Journal of Ambient Intelligence and Smart Environments, 3(3): 253-269.

[41]Lee, J., Reyes, B. A., McManus, D. D., Mathias, O., Chon, K. H. (2012). Atrial fibrillation detection using a smart phone. In proceedings of the 2012 Annual International Conference of the IEEE Engineering in Medicine and Biology Society (EMBC 2012). Aug.28Sep.1, 2012. San Diego, (pp. 1177-1180).

[42] Grimaldi, D., Kurylyak, Y., Lamonaca, F., Nastro, A. (2011). Photoplethysmography detection by smartphone's videocamera. In proceedings of the 6th International Conference on Intelligent Data Acquisition and Advanced Computing Systems (IEEE IDAACS 2011), Sep. 15-17, 2011. Prague, Czech Republic. (Vol. 1, pp. 488-491).

[43] Heathers, J. A. (2013). Smartphone-enabled pulse rate variability: an alternative methodology for the collection of heart rate variability in psychophysiological research. International Journal of Psychophysiology, 89(3): 297-304.

[44]Gregoski, M. J., Mueller, M., Vertegel, A., Shaporev, A., Jackson, B. B., Frenzel, R. M., Treiber, F. A. (2012). Development and validation of a smartphone heart rate acquisition application for health promotion and wellness telehealth applications. International journal of telemedicine and applications, 2012, 1. Article ID 696324

[45]Lakens, D. (2013). Using a Smartphone to measure heart rate changes during relived happiness and anger. IEEE Transactions on Affective Computing, 5(3): 217-226.

[46]Tyagi, A., Miller, K., Cockburn, M. (2012). e-Health tools for targeting and improving melanoma screening: a review. Journal of skin cancer, 2012, Article ID 437502. 
[47]Bourouis, A., Zerdazi, A., Feham, M., and Bouchachia, A. (2013). M-health: skin disease analysis system using Smartphone's camera. Procedia Computer Science, 19 (2013): 1116 1120

[48]Wadhawan, T., Situ, N., Rui, H., Lancaster, K., Yuan, X., Zouridakis, G. (2011). Implementation of the 7-point checklist for melanoma detection on smart handheld devices. In In proceedings of the 2011 Annual International Conference of the IEEE Engineering in Medicine and Biology Society, (EMBC 2011). Aug. 30- Sep 03, 2011. Boston, MA, USA (pp. 3180-3183).

[49]Bourouis, A., Feham, M., Hossain, M. A., Zhang, L. (2014). An intelligent mobile based decision support system for retinal disease diagnosis. Decision Support Systems, 59(2014): 341-350.

[50]Gurrin, C., Qiu, Z., Hughes, M., Caprani, N., Doherty, A. R., Hodges, S. E., and Smeaton, A. F. (2013). The smartphone as a platform for wearable cameras in health research. American journal of preventive medicine, 44(3): 308-313.

[51] Kochanov, D., Jonas, S., Hamadeh, N., Yalvac, E., Slijp, H., Deserno, T. M. (2014). Urban Positioning Using Smartphone-Based Imaging. In Bildverarbeitung für die Medizin, 2014(): 186-191.

[52]Haché, G., Lemaire, E. D., Baddour, N. (2011). Wearable mobility monitoring using a multimedia smartphone platform. IEEE Transactions on Instrumentation and Measurement, 60(9): 3153-3161.

[53]Tapu, R., Mocanu, B., Bursuc, A., Zaharia, T. (2013). A Smartphone-Based Obstacle Detection and Classification System for Assisting Visually Impaired People. In proceedings of the 2013 IEEE International Conference on Computer Vision Workshops (ICCVW 2013). Dec. 2-8, 2013. Sydney, Australia. (pp. 444-451).

[54]Chen, N. C., Wang, K. C., Chu, H. H., (2012). Listen-to-nose: a low-cost system to record nasal symptoms in daily life. In Proceedings of the 2012 ACM Conference on Ubiquitous Computing (UBIComp 2012). Sep. 05 - 08, 2012. Pittsburgh, USA. (pp. 590-591)

[55]Alqassim, S., Ganesh, M., Khoja, S., Zaidi, M., Aloul, F., Sagahyroon, A., (2012), Sleep apnea monitoring using mobile phones. In proceedings of the 14th International Conference on e-Health Networking, Applications and Services (Healthcom 2012). Oct. 10 - 13, 2012. Beijing, China. (pp. 443-446)

[56]Larson, E. C., Goel, M., Boriello, G., Heltshe, S., Rosenfeld, M., and Patel, S. N., (2012), Spirosmart: Using a microphone to measure lung function on a mobile phone, In proceedings of the 2012 ACM Conference on Ubiquitous Computing (UBIComp 2012). Sep. 05 - 08, 2012. Pittsburgh, USA. (pp. 280-289)

[57]Franco, C., Fleury, A., Guméry, P. Y., Diot, B., Demongeot, J., Vuillerme, N., (2013), iBalance-ABF: a smartphone-based audio-biofeedback balance system. IEEE Transactions on Biomedical Engineering, 60( 1):211-215.

[58]Lu, H., Frauendorfer, D., Rabbi, M., Mast, M. S., Chittaranjan, G. T., Campbell, A. T., GaticaPerez , D., Choudhury, T. (2012), StressSense: Detecting stress in unconstrained acoustic 
environments using smartphones.In Proceedings of the 2012 ACM Conference on Ubiquitous Computing (UBIComp 2012). Sep. 05 - 08, 2012. Pittsburgh, USA. (pp. 351-360)

[59]Mosa, A. S. M., Yoo, I., and Sheets, L. (2012). A systematic review of healthcare applications for smartphones. BMC medical informatics and decision making, 12(1): 67.

[60]MedCam App. In Google Play website. Available at:

https://play.google.com/store/apps/details?id=com.cupel.MedCam. [Last access $30 / 11 / 2014]$

[61]Color Detector App In Google Play website. Available at:

//play.google.com/store/apps/details?id=com.mobialia.colordetector. [Last access $30 / 11 / 2014]$

[62]Colorblind Assitant App. In Google Play website. Available at: https://play.google.com/store/apps/details?id=com.unclechromedome.colorblindassistant . [Last access 30/11/2014]

[63]The vOICe for Android App. In Google Play website. Available at: https://play.google.com/store/apps/details?id=vOICe.vOICe. Last aces 30/11/2014]

[64]Voice Cam for Blind App. In Google Play website. Available at:

https://play.google.com/store/apps/details?id=com.prod.voice.cam [Last aces 30/11/2014]

[65]Cardiograph App. In Google Play website. Available at:

https://play.google.com/store/apps/details?id=com.macropinch.hydra.android. [Last access 30/11/2014]

[66] MyEarDroid App. In Google Play website. Available at:

https://play.google.com/store/apps/details?id=com.tecnalia.health.myeardroid. [Last access 30/11/2014]

[67]Quit Snoring App. In Google Play website. Available at:

https://play.google.com/store/apps/details?id=com.ptech_hm.qs. [Last access 30/11/2014

[68]SnoreClock App. In Google Play website. Available at:

https://play.google.com/store/apps/details?id=de.ralphsapps.snorecontrol [Last access 30/11/2014]

[69]Breath Counter App. In Google Play website. Available at:

https://play.google.com/store/apps/details?id=com.softrove.app.bc [Last access 30/11/2014]

[70]Lloret, J., Garcia, M., Bri, D., Diaz, J.R., (2009) A cluster-based architecture to structure the topology of parallel wireless sensor networks, Sensors (Basel). 9(12):10513-10544. 